\title{
SENSITIVE DEPENDENCE OF GEOMETRIC GIBBS STATES AT POSITIVE TEMPERATURE
}

\author{
DANIEL CORONEL AND JUAN RIVERA-LETELIER
}

\begin{abstract}
We give the first example of a smooth family of real and complex maps having sensitive dependence of geometric Gibbs states at positive temperature. This family consists of quadratic-like maps that are non-uniformly hyperbolic in a strong sense. We show that for a dense set of maps in the family the geometric Gibbs states do not converge at positive temperature. These are the first examples of non-convergence at positive temperature in statistical mechanics or the thermodynamic formalism, and answers a question of van Enter and Ruszel. We also show that this phenomenon is robust: There is an open set of analytic 2-parameter families of quadratic-like maps that exhibit sensitive dependence of geometric Gibbs states at positive temperature.
\end{abstract}

\section{INTRODUCTION}

The main problem in statistical mechanics and the thermodynamic formalism is to describe the set of Gibbs states for a given interaction or potential. When the interaction or potential depends on some parameters, one is interested in knowing how the set of Gibbs states changes as a function of these parameters. In several natural models in statistical mechanics, Gibbs states depend continuously (in the weak* topology) on the temperature, even if the number of Gibbs states changes with the temperature, see for instance ADCS15] and references therein. In the thermodynamic formalism, it is known that if the dynamical system has sufficient expansion and the potential is sufficiently regular then in good situations for every value of the temperature there is a unique Gibbs state that varies continuously with the potential, see for example [Bow75, BCFT17, Cli18, Klo17, LRL14a, LRL14b, Prz18, PRL11, PR14, VV10, and references therein.

In this article we study the temperature dependence of Gibbs states at positive temperature of smooth maps for the corresponding geometric potentials. The geometric potential is completely determined by the map. It arises naturally in several important problems, like in the construction of physical measures, as in the pioneering work of Sinaı [Sin72, Ruelle [Rue76, and Bowen Bow75. The pressure of the geometric potential is connected, among other things, to several multifractal spectra, and large deviations rate functions, see for example [BMS03, Lemma 2], CRT16, GPR10, GPR16], KN92, Theorems 1.2 and 1.3], [PRL11, Appendix B], OW17 and references therein.

The presence of a critical point may prevent the system to have geometric Gibbs states. For example, in [CRL13] and [CRL15a] we constructed examples of ColletEckmann maps in the quadratic family, such that for every temperature above a critical value there a unique geometric Gibbs state, and for every temperature smaller than the critical value there is no geometric Gibbs state. However, in those 
examples the geometric Gibbs states converge to a limit measure as the temperature drops to the critical value.

The main result of this article shows that there are analytic families of quadraticlike maps for which an arbitrarily small perturbation of the parameter can have a large effect on the positive-temperature geometric Gibbs states. Furthermore, this phenomenon is robust: There is an open set of analytic 2-parameter families of quadratic-like maps that exhibit "sensitive dependence" of geometric Gibbs states. In particular, this provides the first example in statistical mechanics and the thermodynamic formalism of non-convergence of Gibbs states at positive temperature, thus answering a question raised by van Enter and Ruszel in [vER07. Analogous examples at zero temperature were given in [CR17, see also [BGT18, CH10, CRL15b, vER07] for examples of non-convergence in a symbolic setting.

To state our results more precisely, we recall the concept of quadratic-like maps of Douady and Hubbard [DH85]. Given simply connected subsets $U$ and $V$ of $\mathbb{C}$ satisfying $\operatorname{cl}(U) \subset V$, a holomorphic map $f: U \rightarrow V$ is a quadratic-like map if it is proper of degree 2. Such a map has a unique point at which the derivative $D f$ vanishes; it is the critical point of $f$. The filled-in Julia set of a quadratic-like map $f: U \rightarrow V$ is

$$
K(f):=\left\{z \in U \mid \text { for every integer } n \geq 1, f^{n}(z) \in U\right\} .
$$

The Julia set $J(f)$ of $f$ is the boundary of $K(f)$, and it coincides with the closure of the repelling periodic points of $f$.

Given a quadratic-like map $f$, denote by $\mathscr{M}_{f}$ the space of all probability measures on $J(f)$ that are invariant by $f$. For $\mu$ in $\mathscr{M}_{f}$ denote by $h_{\mu}(f)$ the measure-theoretic entropy of $\mu$, and for each $t$ in $\mathbb{R}$ put

$$
P_{f}(t):=\sup \left\{h_{\mu}(f)-t \int \log |D f| \mathrm{d} \mu \mid \mu \in \mathscr{M}_{f}\right\} .
$$

It is the pressure of $\left.f\right|_{J(f)}$ for the potential $-t \log |D f|$. A measure $\mu$ realizing the supremum above is an equilibrium state of $\left.f\right|_{J(f)}$ for the potential $-t \log |D f|$ or a geometric Gibbs state.

A quadratic-like map $f: U \rightarrow V$ is real if $U, V$, and $f$ are all invariant under complex conjugation. Note that the critical point of such a map is real. A real quadratic-like map with critical point $c$ is essentially topologically exact if $f^{2}(c)$ is defined and is different from $f(c)$, if $f$ maps the interval $I(f)$ bounded by $f(c)$ and $f^{2}(c)$ to itself, and if $\left.f\right|_{I(f)}$ is topologically exact. For such a map $f$ we consider both the interval map $\left.f\right|_{I(f)}$ and the complex map $f$ acting on its Julia set $J(f)$.

Let $f$ be a real quadratic-like map that is essentially topologically exact. Denote by $\mathscr{M}_{f}^{\mathbb{R}}$ the space of all probability measures on $I(f)$ that are invariant by $f$. For $\mu$ in $\mathscr{M}_{f}^{\mathbb{R}}$ we denote by $h_{\mu}(f)$ the measure-theoretic entropy of $\mu$, and for each $t$ in $\mathbb{R}$ we put

$$
P_{f}^{\mathbb{R}}(t):=\sup \left\{h_{\mu}(f)-t \int \log |D f| \mathrm{d} \mu \mid \mu \in \mathscr{M}_{f}^{\mathbb{R}}\right\} .
$$

It is the pressure of $\left.f\right|_{I(f)}$ for the potential $-t \log |D f|$. A measure $\mu$ realizing the supremum above is an equilibrium state of $\left.f\right|_{I(f)}$ for the potential $-t \log |D f|$ or a geometric Gibbs state.

*In $\S 3.1$ we extend the definition of essentially topologically exact map to certain quadratic-like maps that are not necessarily real. 
Definition 1.1 (Sensitive dependence of positive-temperature Gibbs states). Let $\Lambda$ be a topological space and $\left(f_{\lambda}\right)_{\lambda \in \Lambda}$ a continuous family of real or complex quadraticlike maps. The family $\left(f_{\lambda}\right)_{\lambda \in \Lambda}$ has sensitive dependence of positive-temperature geometric Gibbs states, if there are a parameter $\lambda_{0}$ and a positive number $t_{*, 0}$ such that for every increasing sequence of positive number $\left(\delta_{\ell}\right)_{\ell \in \mathbb{N}}$ converging to 1 as $\ell \rightarrow+\infty$, there are a parameter $\lambda$ in $\Lambda$ arbitrarily close to $\lambda_{0}$ and a positive number $t_{*}$ arbitrarily close to $t_{*, 0}$ such that the following property holds: For each $\beta \in\left(0, t_{*}\right)$ there is a unique equilibrium state $\rho_{\beta}^{\mathbb{R}}(\lambda)$ of $\left.f\right|_{I\left(f_{\lambda}\right)}\left(\right.$ resp. $\rho_{\beta}(\lambda)$ of $\left.\left.f\right|_{J\left(f_{\lambda}\right)}\right)$ for the potential $-\beta \log \left|D f_{\lambda}\right|$ and if we put for every $\ell \in \mathbb{N}, \beta_{\ell}:=t_{*} \cdot \delta_{\ell}$, then the sequence of equilibrium states $\left(\rho_{\beta_{\ell}}^{\mathbb{R}}(\lambda)\right)_{\ell \in \mathbb{N}}\left(\right.$ resp. $\left.\left(\rho_{\beta_{\ell}}(\lambda)\right)_{\ell \in \mathbb{N}}\right)$ does not converge.

We note that in the above situation the map $\left.f\right|_{I\left(f_{\lambda}\right)}$ (resp. $\left.\left.f\right|_{J\left(f_{\lambda}\right)}\right)$ must have a "phase transition" at $t=t_{*}$ in the statistical mechanics sense, see [PR14, Theorem A] for the real case, and [PRL11, Main Theorem] for the complex case. Recall that for a real number $t_{*}$, the map $\left.f\right|_{I(f)}$ (resp. $\left.f\right|_{J(f)}$ ) has a phase transition at $t=t_{*}$, if the geometric pressure function $P_{f}^{\mathbb{R}}\left(\right.$ resp. $\left.P_{f}\right)$ is not real analytic at $t=t_{*}$.

Our main results are stated as Theorems $\mathrm{A}$ and $\mathrm{B}$ in 3.4 . The following is a simple consequence of these results that is easier to state.

Sensitive Dependence at Positive Temperature. There is an open subset $\Lambda_{0}$ of $\mathbb{C}$ intersecting $\mathbb{R}$, a holomorphic family of quadratic-like maps $\left(\widehat{f}_{\lambda}\right)_{\lambda \in \Lambda_{0}}$ and a compact subset $\Lambda$ of $\Lambda_{0} \cap \mathbb{R}$ such that the following properties hold. For every real parameter $\lambda$ in $\Lambda$ the map $\widehat{f}_{\lambda}$ is real, and the family of real (resp. complex) maps $\left(\widehat{f}_{\lambda}\right)_{\lambda \in \Lambda}$ has sensitive dependence of positive-temperature geometric Gibbs states. Moreover, this sensitive dependence occurs at every parameter in $\Lambda$, in the sense that every parameter can be considered as $\lambda_{0}$ in Definition 1.1.

The family of quadratic-like maps $\left(\widehat{f}_{\lambda}\right)_{\lambda \in \Lambda_{0}}$ that we use to prove the Sensitive Dependence at Positive Temperature is given explicitly in [CR17, §3.3]. The maps in the subfamily $\left(\widehat{f}_{\lambda}\right)_{\lambda \in \Lambda}$ are transitive and non-uniformly hyperbolic in a strong sense. For example, they satisfy the Collet-Eckmann condition with uniform constants, and moreover they have uniform "goodness constants" in the sense of [BBS15, Definition 2.2], $c f$. Proposition A.4. So, the lack of expansion is not responsible for the sensitive dependence of positive-temperature geometric Gibbs states.

We prove that as the temperature drops to $1 / t_{*}$ the geometric Gibbs states of $\left.\widehat{f}_{\lambda}\right|_{I\left(\widehat{f}_{\lambda}\right)}$ (resp. $\left.\left.\widehat{f}_{\lambda}\right|_{J\left(\widehat{f}_{\lambda}\right)}\right)$ oscillate between 2 periodic measures. So, at certain temperatures the geometric Gibbs state is close to one of these periodic measures, and at other temperatures it is close to the other periodic measure, see Theorem B in 8.4

The conclusions of the Sensitive Dependence at Positive Temperature hold for an open set of holomorphic 2-parameter families of quadratic-like maps, see CR17, Remark 3.4]. Thus, for quadratic-like maps, the sensitive dependence of positivetemperature Gibbs states is a robust phenomenon for 2-parameter families.

Note that the Sensitive Dependence at Positive Temperature does not say anything about the behavior of the geometric Gibbs states of $\left.\widehat{f}_{\lambda_{0}}\right|_{I\left(\widehat{f}_{\lambda_{0}}\right)}$ or $\left.\widehat{f}_{\lambda_{0}}\right|_{J\left(\widehat{f}_{\lambda_{0}}\right)}$ for temperatures above $1 / t_{*, 0}$. As explained in [CR17, Remark 3.5], our results show that the parameter $\lambda_{0}$ can be chosen so that the geometric Gibbs states of $\left.\widehat{f}_{\lambda_{0}}\right|_{I\left(\widehat{f}_{\lambda_{0}}\right)}$ 
(resp. $\left.\left.\widehat{f}_{\lambda_{0}}\right|_{J\left(\widehat{f}_{\lambda_{0}}\right)}\right)$ converge as the temperature drops to $1 / t_{*, 0}$, and that $\lambda_{0}$ can be chosen so that they do not converge.

As a direct consequence of Theorem $\mathrm{A}$ in $\$ 3.4$ we have that for every $\lambda$ in $\Lambda$ the map $\widehat{f}_{\lambda}$ has a phase transition for both pressure functions $P_{\widehat{f}_{\lambda}}^{\mathbb{R}}(t)$ and $P_{\widehat{f}_{\lambda}}(t)$. More precisely, we have the following. For a quadratic-like map $f$ with critical point $c$, put

$$
\chi_{\text {crit }}(f):=\liminf _{n \rightarrow+\infty} \frac{1}{n} \log \left|D f^{n}(f(c))\right| .
$$

High-Order Phase Transitions Theorem. Let $\Lambda_{0}, \Lambda$, and $\left(\widehat{f}_{\lambda}\right)_{\lambda \in \Lambda_{0}}$ be as in the statement of the Sensitive Dependence at Positive Temperature. Then there are positive constants $A^{+}, A^{-}, B^{+}$, and $B^{-}$and a real analytic function $t_{*}: \Lambda_{0} \rightarrow$ $(0,+\infty)$ such that for every $\lambda$ in $\Lambda$, we have for every $t \geq t_{*}(\lambda)$

$$
P_{\widehat{f}_{\lambda}}^{\mathbb{R}}(t)=P_{\widehat{f}_{\lambda}}(t)=-t \frac{\chi_{\text {crit }}\left(\widehat{f}_{\lambda}\right)}{2},
$$

and for every $t$ in $\left(0, t_{*}(\lambda)\right)$ close to $t_{*}(\lambda)$, we have

$$
\begin{aligned}
-t \frac{\chi_{\text {crit }}\left(\widehat{f_{\lambda}}\right)}{2}+2^{-\left(\frac{A^{-}}{\sqrt{t_{*}(\lambda)-t}}+B^{-}\right)^{3}} \leq P_{\widehat{f}_{\lambda}}^{\mathbb{R}}(t) & \leq P_{\widehat{f}_{\lambda}}(t) \\
& \leq-t \frac{\chi_{\text {crit }}\left(\widehat{f}_{\lambda}\right)}{2}+2^{-\left(\frac{A^{+}}{\sqrt{t_{*}(\lambda)-t}}-B^{+}\right)^{3}} .
\end{aligned}
$$

In particular, both $P_{\widehat{f}_{\lambda}}^{\mathbb{R}}$ and $P_{\widehat{f}_{\lambda}}$ are of class $C^{2}$ at $t=t_{*}$, but neither of these functions is real analytic at $t=t_{*}(\lambda)$.

These phase transitions resemble a Kosterlitz-Thouless singularity, see for example [Her07, §6] for background. In fact, near the critical parameter the geometric pressure function behaves as $x \mapsto \exp \left(-|x|^{-3 / 2}\right)$ near $x=0$. The phase transitions in CRL15a, Main Theorem] are similar, but different: The geometric pressure function behaves as $x \mapsto \exp \left(-x^{-2}\right)$ near $x=0$.

The phase transitions in the theorem above are of "freezing type", in the sense that after the phase transition the pressure is affine and equal to its asymptote. Two recent examples of freezing phase transitions in a symbolic setting are in BL13, BL15. The authors prove that above a critical temperature there is a unique Gibbs state with full support and that below the critical temperature the unique Gibbs state is the unique shift-invariant measure of a substitution subshift, like the Thue-Morse subshift. In contrast, in the theorem above for every $\lambda$ in $\Lambda$ and $t \geq t_{*}(\lambda)$ there are no geometric Gibbs state of $\left.\widehat{f}_{\lambda}\right|_{I\left(\widehat{f}_{\lambda}\right)}$ (resp. $\left.\left.\widehat{f}_{\lambda}\right|_{J\left(\widehat{f}_{\lambda}\right)}\right)$ for the potential $-t \log \left|D \widehat{f}_{\lambda}\right|$, see Theorem $\mathrm{A}$ in $\S 3$. Note in particular that, as temperature drops to $1 / t_{*}(\lambda)$, none of the accumulation measures of the geometric Gibbs states is a Gibbs state. See [BTT17, CRL13, DGR14, Vel17] and references therein for other examples of phase transitions in the thermodynamic formalism.

1.1. Organization. After recalling some basic notions of the quadratic family in $\$ 2$ in $\$ 3$ we state Theorems $\mathrm{A}$ and $\mathrm{B}$, and prove the Sensitive Dependence at Positive Temperature assuming these results. To state Theorems $\mathrm{A}$ and $\mathrm{B}$ we recall in $\$ 3.1$ the notion of "uniform families" of quadratic-like maps introduced in [CR17, some general facts about conformal measures in 33.2, and in 33.3 the inducing scheme that was first introduced in CRL13. 
The proof of Theorems $\mathrm{A}$ and $\mathrm{B}$ are given in 4 . After describing the subfamily of maps in 4.1 we give the proof of Theorem $\mathrm{A}$ in 4.2 . It relies on several results and concepts from CR17, including the Geometric Peierls Condition, that we briefly summarize in Appendix $\mathrm{A}$ for the reader's convenience. These results are used to estimate the pressure and conformal measures in terms of the postcritical series. The proof of Theorem $\mathrm{B}$ is given in $\$ 4.3$ Roughly speaking, the most difficult part is to estimate the postcritical series by a certain numerical series. The necessary estimates of the numerical series, in Appendix B, are given in an abstract setting that is independent of the rest of the paper.

1.2. Notes and references. The proofs of Theorems $\mathrm{A}$ and $\mathrm{B}$ rely strongly on estimates for the pressure functions and conformal measures for quasi-quadratic maps satisfying the Geometric Peierls Condition proved in [CR17. See Appendix A for the definition of this concept. Indeed, we use the same family of quasi-quadratic maps $\left(\widehat{f}_{\lambda}\right)_{\lambda \in \Lambda_{0}}$ as in [CR17] but here we choose a different set of parameters. That is, we choose a different subset $\Lambda$ of $\Lambda_{0}$.

1.3. Acknowledgments. We would like to thank the referees for the comments and pointers to the literature.

The first named author acknowledges partial support from FONDECYT grant 1161221, and would like to thank the University of Rochester for its hospitality. The second named author acknowledges partial support from NSF grant DMS-1700291, and would like to thank Universidad Andrés Bello for its hospitality.

\section{Preliminaries}

The main objective of this section is to recall the definition of Yoccoz puzzle pieces in 2.4 and to introduce a subset of parameters of the quadratic family in $\$ 2.6$ that possesses the combinatorics needed for our results.

We use $\mathbb{N}$ to denote the set of integers that are greater than or equal to 1 and $\mathbb{N}_{0}:=\mathbb{N} \cup\{0\}$. For a Borel measure $\rho$ on $\mathbb{C}$, denote by $\operatorname{supp}(\rho)$ its support. Given an open subset $G$ of $\mathbb{C}$ and a map $f: G \rightarrow \mathbb{C}$ that is a biholomorphism onto its image, the distortion of $f$ on a subset $C$ of $G$ is

$$
\sup _{x, y \in C} \frac{|D f(x)|}{|D f(y)|} .
$$

2.1. Quadratic polynomials, Green's functions, and Böttcher coordinates. In this subsection and the next we recall some basic facts about the dynamics of complex quadratic polynomials, see for instance [CG93 or Mil06 for references.

For $c$ in $\mathbb{C}$ we denote by $f_{c}$ the complex quadratic polynomial

$$
f_{c}(z)=z^{2}+c,
$$

and by $K_{c}$ the filled Julia set of $f_{c}$; that is, the set of all points $z$ in $\mathbb{C}$ whose forward orbit under $f_{c}$ is bounded in $\mathbb{C}$. The set $K_{c}$ is compact and its complement is the connected set consisting of all points whose orbit converges to infinity in the Riemann sphere. Furthermore, we have $f_{c}^{-1}\left(K_{c}\right)=K_{c}$ and $f_{c}\left(K_{c}\right)=K_{c}$. The boundary $J_{c}$ of $K_{c}$ is the Julia set of $f_{c}$. 
For a parameter $c$ in $\mathbb{C}$, the Green's function of $K_{c}$ is the function $G_{c}: \mathbb{C} \rightarrow$ $[0,+\infty)$ that is identically 0 on $K_{c}$, and that for $z$ outside $K_{c}$ is given by the limit,

$$
G_{c}(z)=\lim _{n \rightarrow+\infty} \frac{1}{2^{n}} \log \left|f_{c}^{n}(z)\right|>0 .
$$

The function $G_{c}$ is continuous, subharmonic, satisfies $G_{c} \circ f_{c}=2 G_{c}$ on $\mathbb{C}$, and it is harmonic and strictly positive outside $K_{c}$. On the other hand, the critical values of $G_{c}$ are bounded from above by $G_{c}(0)$, and the open set

$$
U_{c}:=\left\{z \in \mathbb{C} \mid G_{c}(z)>G_{c}(0)\right\}
$$

is homeomorphic to a punctured disk. Notice that $G_{c}(c)=2 G_{c}(0)$, thus $U_{c}$ contains $c$ if $0 \notin K_{c}$.

By Böttcher's Theorem there is a unique conformal representation

$$
\varphi_{c}: U_{c} \rightarrow\left\{z \in \mathbb{C}|| z \mid>\exp \left(G_{c}(0)\right)\right\},
$$

and this map conjugates $f_{c}$ to $z \mapsto z^{2}$. It is called the Böttcher coordinate of $f_{c}$ and satisfies $G_{c}=\log \left|\varphi_{c}\right|$.

2.2. External rays and equipotentials. Let $c$ be in $\mathbb{C}$. For $v>0$ the equipotential $v$ of $f_{c}$ is by definition $G_{c}^{-1}(v)$. A Green's line of $G_{c}$ is a smooth curve on the complement of $K_{c}$ in $\mathbb{C}$ that is orthogonal to the equipotentials of $G_{c}$ and that is maximal with this property. Given $t$ in $\mathbb{R} / \mathbb{Z}$, the external ray of angle $t$ of $f_{c}$, denoted by $R_{c}(t)$, is the Green's line of $G_{c}$ containing

$$
\left\{\varphi_{c}^{-1}(r \exp (2 \pi i t)) \mid \exp \left(G_{c}(0)\right)<r<+\infty\right\} .
$$

By the identity $G_{c} \circ f_{c}=2 G_{c}$, for each $v>0$ and each $t$ in $\mathbb{R} / \mathbb{Z}$ the map $f_{c}$ maps the equipotential $v$ to the equipotential $2 v$ and maps $R_{c}(t)$ to $R_{c}(2 t)$. For $t$ in $\mathbb{R} / \mathbb{Z}$ the external ray $R_{c}(t)$ lands at a point $z$, if $G_{c}: R_{c}(t) \rightarrow(0,+\infty)$ is a bijection and if $\left.G_{c}\right|_{R_{c}(t)} ^{-1}(v)$ converges to $z$ as $v$ converges to 0 in $(0,+\infty)$. By the continuity of $G_{c}$, every landing point is in $J_{c}=\partial K_{c}$.

The Mandelbrot set $\mathcal{M}$ is the subset of $\mathbb{C}$ of those parameters $c$ for which $K_{c}$ is connected. The function

$$
\begin{aligned}
\Phi: \mathbb{C} \backslash \mathcal{M} & \rightarrow \mathbb{C} \backslash \operatorname{cl}(\mathbb{D}) \\
c & \mapsto \Phi(c):=\varphi_{c}(c)
\end{aligned}
$$

is a conformal representation, see [DH84, VIII, Théorème 1]. For $v>0$ the equipotential $v$ of $\mathcal{M}$ is by definition

$$
\mathcal{E}(v):=\Phi^{-1}(\{z \in \mathbb{C}|| z \mid=v\}) .
$$

On the other hand, for $t$ in $\mathbb{R} / \mathbb{Z}$ the set

$$
\mathcal{R}(t):=\Phi^{-1}(\{r \exp (2 \pi i t) \mid r>1\})
$$

is called the external ray of angle $t$ of $\mathcal{M}$. We say that $\mathcal{R}(t)$ lands at a point $z$ in $\mathbb{C}$, if $\Phi^{-1}(r \exp (2 \pi i t))$ converges to $z$ as $r \searrow 1$. When this happens $z$ belongs to $\partial \mathcal{M}$. 
2.3. The wake $1 / 2$. In this subsection we recall a few facts that can be found for example in [DH84] or [Mil00].

The external rays $\mathcal{R}(1 / 3)$ and $\mathcal{R}(2 / 3)$ of $\mathcal{M}$ land at the parameter $c=-3 / 4$, and these are the only external rays of $\mathcal{M}$ that land at this point, see for example [Mil00, Theorem 1.2]. In particular, the complement in $\mathbb{C}$ of the set

$$
\mathcal{R}(1 / 3) \cup \mathcal{R}(2 / 3) \cup\{-3 / 4\}
$$

has 2 connected components; we denote by $\mathcal{W}$ the connected component containing the point $c=-2$ of $\mathcal{M}$.

For each parameter $c$ in $\mathcal{W}$ the map $f_{c}$ has 2 distinct fixed points; one of the them is the landing point of the external ray $R_{c}(0)$ and it is denoted by $\beta(c)$; the other one is denoted by $\alpha(c)$. The only external ray landing at $\beta(c)$ is $R_{c}(0)$, and the only external ray landing at $-\beta(c)$ is $R_{c}(1 / 2)$.

Moreover, for every parameter $c$ in $\mathcal{W}$ the only external rays of $f_{c}$ landing at $\alpha(c)$ are $R_{c}(1 / 3)$ and $R_{c}(2 / 3)$, see for example [Mil00, Theorem 1.2]. The complement of $R_{c}(1 / 3) \cup R_{c}(2 / 3) \cup\{\alpha(c)\}$ in $\mathbb{C}$ has 2 connected components; one containing $-\beta(c)$ and $z=c$, and the other one containing $\beta(c)$ and $z=0$. On the other hand, the point $\alpha(c)$ has 2 preimages by $f_{c}$ : Itself and $\widetilde{\alpha}(c):=-\alpha(c)$. The only external rays landing at $\widetilde{\alpha}(c)$ are $R_{c}(1 / 6)$ and $R_{c}(5 / 6)$.

2.4. Yoccoz puzzles and para-puzzle. In this subsection we recall the definitions of Yoccoz puzzles and para-puzzle. We follow [Roe00].

Definition 2.1 (Yoccoz puzzles). Fix $c$ in $\mathcal{W}$ and consider the open region $X_{c}^{\circ}:=$ $\left\{z \in \mathbb{C} \mid G_{c}(z)<1\right\}$. The Yoccoz puzzle of $f_{c}$ is given by the following sequence of graphs $\left(I_{c, n}\right)_{n=0}^{+\infty}$ defined for $n=0$ by:

$$
I_{c, 0}:=\partial X_{c}^{\circ} \cup\left(X_{c}^{\circ} \cap \operatorname{cl}\left(R_{c}(1 / 3)\right) \cap \operatorname{cl}\left(R_{c}(2 / 3)\right)\right),
$$

and for $n \geq 1$ by $I_{c, n}:=f_{c}^{-n}\left(I_{c, 0}\right)$. The puzzle pieces of depth $n$ are the connected components of $f_{c}^{-n}\left(X_{c}^{\circ}\right) \backslash I_{c, n}$. The puzzle piece of depth $n$ containing a point $z$ is denoted by $P_{c, n}(z)$.

Note that for a real parameter $c$, every puzzle piece intersecting the real line is invariant under complex conjugation. Since puzzle pieces are simply-connected, it follows that the intersection of such a puzzle piece with $\mathbb{R}$ is an interval.

Definition 2.2 (Yoccoz para-puzzle甲). Given an integer $n \geq 0$, put

$$
J_{n}:=\left\{t \in[1 / 3,2 / 3] \mid 2^{n} t(\bmod 1) \in\{1 / 3,2 / 3\}\right\},
$$

let $\mathcal{X}_{n}$ be the intersection of $\mathcal{W}$ with the open region in the parameter plane bounded by the equipotential $\mathcal{E}\left(2^{-n}\right)$ of $\mathcal{M}$, and put

$$
\mathcal{I}_{n}:=\partial \mathcal{X}_{n} \cup\left(\mathcal{X}_{n} \cap \bigcup_{t \in J_{n}} \operatorname{cl}(\mathcal{R}(t))\right)
$$

Then the Yoccoz para-puzzle of $\mathcal{W}$ is the sequence of graphs $\left(\mathcal{I}_{n}\right)_{n=0}^{+\infty}$. The parapuzzle pieces of depth $n$ are the connected components of $\mathcal{X}_{n} \backslash \mathcal{I}_{n}$. The para-puzzle piece of depth $n$ containing a parameter $c$ is denoted by $\mathcal{P}_{n}(c)$.

\footnotetext{
${ }^{\dagger}$ In contrast to Roe00, we only consider the para-puzzle in the wake $\mathcal{W}$.
} 
Observe that there is only 1 para-puzzle piece of depth 0 , and only 1 parapuzzle piece of depth 1; they are bounded by the same external rays but different equipotentials. Both of them contain $c=-2$.

Fix a parameter $c$ in $\mathcal{P}_{0}(-2)$. There are precisely 2 puzzle pieces of depth 0 : $P_{c, 0}(\beta(c))$ and $P_{c, 0}(-\beta(c))$. Each of them is bounded by the equipotential 1 and by the closures of the external rays landing at $\alpha(c)$. Furthermore, the critical value $c$ of $f_{c}$ is contained in $P_{c, 0}(-\beta(c))$ and the critical point in $P_{c, 0}(\beta(c))$. It follows that the set $f_{c}^{-1}\left(P_{c, 0}(\beta(c))\right)$ is the disjoint union of $P_{c, 1}(-\beta(c))$ and $P_{c, 1}(\beta(c))$, so $f_{c}$ maps each of the sets $P_{c, 1}(-\beta(c))$ and $P_{c, 1}(\beta(c))$ biholomorphically to $P_{c, 0}(\beta(c))$. Moreover, there are precisely 3 puzzle pieces of depth 1 :

$$
P_{c, 1}(-\beta(c)), P_{c, 1}(0) \quad \text { and } \quad P_{c, 1}(\beta(c)) ;
$$

$P_{c, 1}(-\beta(c))$ is bounded by the equipotential $1 / 2$ and by the closures of the external rays that land at $\alpha(c) ; P_{c, 1}(\beta(c))$ is bounded by the equipotential $1 / 2$ and by the closures of the external rays that land at $\widetilde{\alpha}(c)$; and $P_{c, 1}(0)$ is bounded by the equipotential $1 / 2$ and by the closures of the external rays that land at $\alpha(c)$ and at $\widetilde{\alpha}(c)$. In particular, the closure of $P_{c, 1}(\beta(c))$ is contained in $P_{c, 0}(\beta(c))$. It follows from this that for each integer $n \geq 1$ the map $f_{c}^{n}$ maps $P_{c, n}(-\beta(c))$ biholomorphically to $P_{c, 0}(\beta(c))$.

The following lemma proved in [CRL13, Lemma 3.3] is useful for the description of the parameter introduced in $\$ \$ 2.6$

Lemma 2.3. For each integer $n \geq 1$, the following properties hold.

1. The para-puzzle piece $\mathcal{P}_{n}(-2)$ contains the closure of $\mathcal{P}_{n+1}(-2)$.

2. For each parameter $c$ in $\mathcal{P}_{n}(-2)$ the critical value $c$ of $f_{c}$ is in $P_{c, n}(-\beta(c))$.

2.5. The uniformly expanding Cantor set. For a parameter $c$ in $\mathcal{P}_{3}(-2)$, the maximal invariant set $\Lambda_{c}$ of $f_{c}^{3}$ in $P_{c, 1}(0)$ plays an important role in the definition of the combinatorics of the quadratic-like maps used in the proof of Theorem B. We recall here its definition using certain puzzle pieces that we also use in the next section.

Fix $c$ in $\mathcal{P}_{3}(-2)$. There are precisely 2 connected components of $f_{c}^{-3}\left(P_{c, 1}(0)\right)$ contained in $P_{c, 1}(0)$ that we denote by $Y_{c}$ and $\tilde{Y}_{c}$. The closures of these sets are disjoint and contained in $P_{c, 1}(0)$. The sets $Y_{c}$ and $\tilde{Y}_{c}$ are distinguished by the fact that $Y_{c}$ contains in its boundary the common landing point of the external rays $R_{c}(7 / 24)$ and $R_{c}(17 / 24)$, denoted $\gamma(c)$, and that $\tilde{Y}_{c}$ contains in its boundary the common landing point of the external rays $R_{c}(5 / 24)$ and $R_{c}(19 / 24)$. The map $f_{c}^{3}$ maps each of the sets $Y_{c}$ and $\widetilde{Y}_{c}$ biholomorphically to $P_{c, 1}(0)$. Thus, if we put

$$
\begin{aligned}
g_{c}: Y_{c} \cup \tilde{Y}_{c} & \rightarrow P_{c, 1}(0) \\
z & \mapsto g_{c}(z):=f_{c}^{3}(z),
\end{aligned}
$$

then

$$
\Lambda_{c}=\bigcap_{n \in \mathbb{N}} g_{c}^{-n}\left(\operatorname{cl}\left(P_{c, 1}(0)\right)\right) .
$$

2.6. Parameters. The combinatorics of the quadratic-like maps that we use to prove Theorem $\mathrm{B}$ are modeled from a subset of the quadratic family introduced in [CRL13, Proposition 3.1]. In this subsection we recall the definition of this parameter set. 
Given an integer $n \geq 3$, let $\mathcal{K}_{n}$ be the set of all those real parameters $c<0$ such that

$$
f_{c}(c)>f_{c}^{2}(c)>\cdots>f_{c}^{n-1}(c)>0 \quad \text { and } \quad f_{c}^{n}(c) \in \Lambda_{c} .
$$

Note that for a parameter $c$ in $\mathcal{K}_{n}$, the critical point of $f_{c}$ cannot be asymptotic to a non-repelling periodic point. This implies that all the periodic points of $f_{c}$ in $\mathbb{C}$ are hyperbolic repelling and therefore that $K_{c}=J_{c}$, see [Mil06. On the other hand, we have $f_{c}(c)>c$ and the interval $I_{c}=\left[c, f_{c}(c)\right]$ is invariant by $f_{c}$. This implies that $I_{c}$ is contained in $J_{c}$ and hence that for every real number $t$ we have $P_{c}^{\mathbb{R}}(t) \leq P_{c}(t)$. Note also that $\left.f_{c}\right|_{I_{c}}$ is not renormalizable, so $f_{c}$ is topologically exact on $I_{c}$, see for example [MvS93, Theorem III.4.1].

Since for $c$ in $\mathcal{K}_{n}$ the critical point of $f_{c}$ is not periodic, for every integer $k \geq 0$ we have $f_{c}^{n+3 k}(c) \neq 0$. Thus, we can define the sequence $\iota(c)$ in $\{0,1\}^{\mathbb{N}_{0}}$ for each $k \geq 0$ by

$$
\iota(c)_{k}:= \begin{cases}0 & \text { if } f_{c}^{n+3 k}(c) \in Y_{c} \\ 1 & \text { if } f_{c}^{n+3 k}(c) \in \widetilde{Y}_{c} .\end{cases}
$$

The following proposition without the statement about the homeomorphism was proved in CRL13, Proposition 3.1]. For the proof of the homeomorphism property see [CR17, Proposition 2.3].

Proposition 2.4. For each integer $n \geq 3$, the set $\mathcal{K}_{n}$ is a compact subset of

$$
\mathcal{P}_{n}(-2) \cap(-2,-3 / 4),
$$

and the function $\iota: \mathcal{K}_{n} \rightarrow\{0,1\}^{\mathbb{N}_{0}}$ is homeomorphism. Finally, for each $\delta>0$ there is $n_{0} \geq 3$ such that for each integer $n \geq n_{0}$ the set $\mathcal{K}_{n}$ is contained in the interval $(-2,-2+\delta)$.

\section{MAin Results}

In this section we state Theorem B and prove the Sensitive Dependence at Positive Temperature assuming this result. We also state a more precise version of the High-Order Phase Transitions Theorem as Theorem A

In $\$ 3.1$ we recall some generalities about uniform families of quadratic-like maps. In 83.2 we recall a general result about conformal measures and in $\$ 3.3$ the inducing scheme that we use to estimate the pressure function and the conformal measures. Then, we state Theorems $\mathrm{A}$ and $\mathrm{B}$ in $\$ 3.4$, and we assume this last result in $\$ 3.5$ to prove the Sensitive Dependence at Positive Temperature.

3.1. Uniform families of quadratic-like maps. A quadratic-like map $f: U \rightarrow$ $V$ is normalized, if its unique critical point is 0 , and if $D^{2} f(0)=2$. For such a map $f$ there is a holomorphic function $R_{f}: U \rightarrow \mathbb{C}$ such that for $w$ in $U$ we have

$$
f(w)=f(0)+w^{2}+w^{3} R_{f}(w) .
$$

Note that $f$ is uniquely determined by its critical value $f(0)$, and the function $R_{f}$.

By the straightening theorem of Douady and Hubbard [DH85], for every quadraticlike map $f: U \rightarrow V$ there is $c$ in $\mathbb{C}$ and a quasi-conformal homeomorphism $h: \mathbb{C} \rightarrow \mathbb{C}$ that conjugates the quadratic polynomial $f_{c}$ to $f$ on a neighborhood of $J_{c}$. In the case $f$ is real, $c$ is real, and $h$ can be chosen so that it commutes with the complex conjugation. In all the cases, the quasi-conformal homeomorphism $h$ can be chosen to be holomorphic on a neighborhood of infinity, and tangent to the identity there. 
Put

$$
\mathcal{X}:=\left\{c \in \mathbb{C} \mid G_{c}(c) \leq 1\right\} \quad \text { and } \quad \widehat{\mathcal{X}}:=\left\{c \in \mathbb{C} \mid G_{c}(c) \leq 2\right\},
$$

and for $c$ in $\mathbb{C}$, put

$$
X_{c}:=\left\{z \in \mathbb{C} \mid G_{c}(z) \leq 1\right\} \quad \text { and } \quad \widehat{X}_{c}:=\left\{z \in \mathbb{C} \mid G_{c}(z) \leq 2\right\} .
$$

Note that $X_{c}$ is contained in the interior of $\widehat{X}_{c}$, and that

$$
\mathcal{X}=\left\{c \in \mathbb{C} \mid c \in X_{c}\right\} \quad \text { and } \quad \widehat{\mathcal{X}}=\left\{c \in \mathbb{C} \mid c \in \widehat{X}_{c}\right\} .
$$

Definition 3.1 (Uniform family of quadratic-like maps). A family $\mathscr{F}$ of normalized quadratic-like maps is uniform, if there are constants $K \geq 1$ and $R>0$, such that for each $f$ in $\mathscr{F}$ there are $c(f)$ in $\mathcal{X}$ and a $K$-quasi-conformal homeomorphism $h_{f}$ of $\mathbb{C}$ satisfying the following properties.

1. The homeomorphism $h_{f}$ conjugates $f_{c(f)}$ on $\widehat{X}_{c(f)}$ to $f$ on $h_{f}\left(\widehat{X}_{c(f)}\right)$. Furthermore, if $f$ is real, then $h_{f}$ commutes with the complex conjugation.

2. The set $\widehat{X}_{c(f)}$ is contained in $B(0, R)$, and the homeomorphism $h_{f}$ is holomorphic on $\mathbb{C} \backslash \operatorname{cl}(B(0, R))$, and it is tangent to the identity at infinity.

Note that property 1 implies that $h_{f}(0)=0$.

Let $\mathscr{F}$ be a uniform family of quadratic-like maps. For each $f$ in $\mathscr{F}$ put

$$
X_{f}:=h_{f}\left(X_{c(f)}\right) \quad \text { and } \quad \widehat{X}_{f}:=h_{f}\left(\widehat{X}_{c(f)}\right) \text {. }
$$

By the definition of uniform family, the puzzle pieces of $f_{c(f)}$ can be push-forward to $X_{f}$ by $h_{f}$. We call to these sets the puzzle pieces of $f$. We say that a puzzle piece of $f$ has depth $n$ if it is the push-forward of a puzzle piece of $c(f)$ with depth $n$. The puzzle piece of depth $n$ of $f$ containing $w$ is denoted $P_{f, n}(w)$. Thus, we have

$$
P_{f, n}(w):=h_{f}\left(P_{c(f), n}\left(h_{f}^{-1}(w)\right)\right) .
$$

Set

$$
\beta(f):=h_{f}(\beta(c(f))) \text { and } \widetilde{\beta}(f):=h_{f}(-\beta(c(f))) .
$$

For every integer $n \geq 0$, put

$$
\mathcal{P}_{n}(\mathscr{F}):=\left\{f \in \mathscr{F} \mid c(f) \in \mathcal{P}_{n}(-2)\right\}
$$

and for $n \geq 3$, put

$$
\mathcal{K}_{n}(\mathscr{F}):=\left\{f \in \mathscr{F} \mid c(f) \in \mathcal{K}_{n}\right\} .
$$

Moreover, for $f$ in $\mathcal{P}_{3}(\mathscr{F})$ put

$$
Y_{f}:=h_{f}\left(Y_{c(f)}\right) \text {, and } \widetilde{Y}_{f}:=h_{f}\left(\widetilde{Y}_{c(f)}\right) \text {, }
$$

and let $g_{f}: h_{f}\left(Y_{c(f)} \cup \widetilde{Y}_{c(f)}\right) \rightarrow P_{f, 1}(0)$ be defined by $g_{f}:=h_{f}^{-1} \circ g_{c(f)} \circ h_{f}$. Moreover, let $p(f)$ and $p^{+}(f)$ be the unique fixed point of $g_{f}$ in $Y_{f}$ and $\widetilde{Y}_{f}$, respectively, and denote by $p^{-}(f)$ the unique fixed point of $g_{f}^{2}$ in $\widetilde{Y}_{f}$ that is different from $p^{+}(f)$; it is a periodic point of $g_{f}$ of minimal period 2. Furthermore, denote by

$\mathcal{O}^{+}(f):=\left\{f^{j}\left(p^{+}(f)\right) \mid j \in\{0,1,2\}\right\}$ and $\mathcal{O}^{-}(f):=\left\{f^{j}\left(p^{-}(f)\right) \mid j \in\{0,1, \ldots, 5\}\right\}$ the orbits of $p^{+}(f)$ and $p^{-}(f)$ under $f$, respectively.

For each integer $n \geq 5$, and each $f$ in $\mathcal{K}_{n}(\mathscr{F})$, put $\iota(f):=\iota(c(f))$, see $\$ 2.6$, and note that for every integer $j \geq 0$ we have

$$
\iota(f)_{j}:= \begin{cases}0 & \text { if } f^{n+1+3 j}(0) \in Y_{f} \\ 1 & \text { if } f^{n+1+3 j}(0) \in \widetilde{Y}_{f}\end{cases}
$$


Finally, for every $f$ in $\mathscr{F}$ such that $c(f)$ is real and belongs to $[-2,0)$, denote by $I(f)$ the image under $h_{f}$ of the interval $\left[c(f), f_{c(f)}(c(f))\right]$. Observe that $f(I(f))=I(f)$. A quadratic-like map $f$ in $\mathscr{F}$ is essentially topologically exact if $c(f)$ is in $[-2,0)$, and if $\left.f\right|_{I(f)}$ is topologically exact. For such a map $f$ we consider both, the map $\left.f\right|_{I(f)}$, and the complex map $f$ acting on its Julia set $J(f)$. We also define $\mathscr{M}_{f}^{\mathbb{R}}, P_{f}^{\mathbb{R}}$, and equilibrium states or Geometric Gibbs states of $\left.f\right|_{I(f)}$ as in the introduction. In the case $f$ is real, the definitions above coincide with those in the introduction.

3.2. Conformal measures. Throughout this section we fix a uniform family of quadratic-like maps $\mathscr{F}$.

Let $n \geq 4$ be an integer and $f$ in $\mathcal{K}_{n}(\mathscr{F})$. Given $t>0$ and a real number $p$, a measure $\mu$ is $(t, p)$-conformal for $\left.f\right|_{I(f)}$ (resp. $f$ ), if for every subset $U$ of $I(f)$ (resp. $J(f)$ ) on which $\left.f\right|_{I(f)}$ (resp. $f$ ) is injective we have

$$
\mu\left(\left.f\right|_{I(f)}(U)\right)=\exp (p) \int_{U}|D f|^{t} \mathrm{~d} \mu\left(\operatorname{resp} . \mu(f(U))=\exp (p) \int_{U}|D f|^{t} \mathrm{~d} \mu\right) .
$$

In the case where $P_{f}^{\mathbb{R}}(t)=0$ (resp. $\left.P_{f}^{\mathbb{C}}(t)=0\right)$, a $(t, 0)$-conformal measure is simply called conformal.

On the other hand, the conical or radial Julia set of $\left.f\right|_{I(f)}$ (resp. $f$ ) is the set of all points $x$ in $I(f)$ (resp. $J(f)$ ) for which the following property holds: There exists $r>0$ and an unbounded sequence of positive integers $\left(n_{j}\right)_{j=1}^{+\infty}$, such that for every $j$ the map $\left.f\right|_{I(f)} ^{n_{j}}\left(\right.$ resp. $\left.f^{n_{j}}\right)$ maps a neighborhood of $x$ in $I(f)($ resp. $J(f))$ diffeomorphically to $B\left(f^{n_{j}}(x), r\right)$.

In the case where $f$ is a real quadratic map, the following is CRL15a, Proposition 5.1]. The proof applies without change to quadratic-like maps in $\mathcal{K}_{n}(\mathscr{F})$.

Proposition 3.2 (CRL15a, Proposition 5.1). Let $n \geq 4$ be an integer, $f$ a map in $\mathcal{K}_{n}(\mathscr{F})$, and let $t>0$ and $p$ in $\mathbb{R}$ be given. Then there is at most one $(t, p)$-conformal probability measure of $\left.f\right|_{I(f)}($ resp. $f)$ supported on $I(f)$ (resp. $\left.J(f)\right)$. If such a measure $\mu$ exists, then $p \geq P_{f}^{\mathbb{R}}(t)$ (resp. $p \geq P_{f}^{\mathbb{C}}(t)$ ), and $\mu$ is either supported on the backward orbit of $z=0$ and dissipative, or $\mu$ is nonatomic and supported on the conical Julia set of $\left.f\right|_{I(f)}$ (resp. $f$ ). Furthermore, the former case holds precisely when the following series converges:

$$
\begin{aligned}
\sum_{j=1}^{+\infty} \exp (-j p) \sum_{\left.y \in f\right|_{I(f)} ^{-j}(0)}\left|D f^{j}(y)\right|^{-t} & \\
& \left(\text { resp. } \sum_{j=1}^{+\infty} \exp (-j p) \sum_{y \in f^{-j}(0)}\left|D f^{j}(y)\right|^{-t}\right) .
\end{aligned}
$$

3.3. Inducing scheme and conformal measures. Throughout this subsection we fix a uniform family of quadratic-like maps $\mathscr{F}$. In this subsection we introduce the inducing scheme to estimate the geometric pressure function for maps in $\mathcal{K}_{n}(\mathscr{F})$.

Let $n \geq 5$ be an integer and $f$ in $\mathcal{K}_{n}(\mathscr{F})$. Put

$$
V_{f}:=P_{f, n+1}(0)=f^{-1}\left(P_{f, n}(\widetilde{\beta}(f))\right)
$$

and

$$
D_{f}:=\left\{z \in V_{f} \mid f^{m}(z) \in V_{f} \text { for some } m \geq 1\right\} .
$$


For $w$ in $D_{f}$ put $m_{f}(w):=\min \left\{m \in \mathbb{N} \mid f^{m}(w) \in V_{f}\right\}$, and call it the first return time of $w$ to $V_{f}$. The first return map to $V_{f}$ is defined by

$$
\begin{aligned}
F_{f}: D_{f} & \rightarrow V_{f} \\
w & \mapsto F_{f}(w):=f^{m_{f}(w)}(w) .
\end{aligned}
$$

It is easy to see that $D_{f}$ is a disjoint union of puzzle pieces; so each connected component of $D_{f}$ is a puzzle piece. Note furthermore that in each of these puzzle pieces $W$, the return time function $m_{f}$ is constant; denote the common value of $m_{f}$ on $W$ by $m_{f}(W)$.

Throughout the rest of this subsection we put $\widehat{V}_{f}:=P_{f, 4}(0)$.

Lemma 3.3 (Uniform distortion bound, CR17, Lemma 5.1). There is a constant $\Xi_{1}>1$ such that for each integer $n \geq 5$ and each $f$ in $\mathcal{K}_{n}(\mathscr{F})$ the following property holds: For every connected component $W$ of $D_{f}$ the map $\left.F_{f}\right|_{W}$ is univalent and its distortion is bounded by $\Xi_{1}$. Furthermore, the inverse of $\left.F_{f}\right|_{W}$ admits a univalent extension to $\widehat{V}_{f}$ taking images in $V_{f}$. In particular, $F_{f}$ is uniformly expanding with respect to the hyperbolic metric on $\widehat{V}_{f}$.

Denote by $\mathfrak{D}_{f}$ the collection of connected components of $D_{f}$ and if $c(f)$ is real denote by $\mathfrak{D}_{f}^{\mathbb{R}}$ the sub-collection of $\mathfrak{D}_{f}$ of those sets intersecting $I(f)$. For each $W$ in $\mathfrak{D}_{f}$ denote by $\phi_{W}: \widehat{V}_{f} \rightarrow V_{f}$ the extension of $\left.f\right|_{W} ^{-1}$ given by Lemma 3.3. Given an integer $\ell \geq 1$ we denote by $E_{f, \ell}$ (resp. $E_{f, \ell}^{\mathbb{R}}$ ) the set of all words of length $\ell$ in the alphabet $\mathfrak{D}_{f}\left(\right.$ resp. $\left.\mathfrak{D}_{f}^{\mathbb{R}}\right)$. Again by Lemma 3.3, for each integer $\ell \geq 1$ and each word $W_{1} \cdots W_{\ell}$ in $E_{f, \ell}$ the composition

$$
\phi_{W_{1} \cdots W_{\ell}}=\phi_{W_{1}} \circ \cdots \circ \phi_{W_{\ell}}
$$

is defined on $\widehat{V}_{f}$. We also put

$$
m_{f}\left(W_{1} \cdots W_{\ell}\right)=m_{f}\left(W_{1}\right)+\cdots+m_{f}\left(W_{\ell}\right) .
$$

For $t, p$ in $\mathbb{R}$ and an integer $\ell \geq 1$ put

$$
Z_{\ell}(t, p):=\sum_{\underline{W} \in E_{f, \ell}} \exp \left(-m_{f}(\underline{W}) p\right)\left(\sup \left\{\left|D \phi_{\underline{W}}(z)\right| \mid z \in V_{f}\right\}\right)^{t}
$$

and

$$
Z_{\ell}^{\mathbb{R}}(t, p):=\sum_{\underline{W} \in E_{f, \ell}^{\mathbb{R}}} \exp \left(-m_{f}(\underline{W}) p\right)\left(\sup \left\{\left|D \phi_{\underline{W}}(z)\right| \mid z \in V_{f}\right\}\right)^{t} .
$$

For a fixed $t$ and $p$ in $\mathbb{R}$ the sequence

$$
\left(\frac{1}{\ell} \log Z_{\ell}(t, p)\right)_{\ell=1}^{+\infty}\left(\operatorname{resp} \cdot\left(\frac{1}{\ell} \log Z_{\ell}^{\mathbb{R}}(t, p)\right)_{\ell=1}^{+\infty}\right)
$$

converges to the pressure function of $F_{f}$ (resp. $\left.\left.F_{f}\right|_{D_{f} \cap I(f)}\right)$ for the potential $-t \log \left|D F_{f}\right|-$ $p m_{f}$; we denote it by $\mathscr{P}_{f}(t, p)$ (resp. $\left.\mathscr{P}_{f}^{\mathbb{R}}(t, p)\right)$. On the set where it is finite, the function $\mathscr{P}_{f}$ (resp. $\mathscr{P}_{f}^{\mathbb{R}}$ ) so defined is strictly decreasing in each of its variables.

Given $t>0$ and $p$ in $\mathbb{R}$, a finite measure $\widetilde{\mu}$ on $\mathbb{C}$ that is supported on the maximal invariant set of $\left.F_{f}\right|_{D_{f} \cap \mathbb{R}}$ (resp. $\left.F_{f}\right)$ is $(t, p)$-conformal for $F_{f}$, if for every $W$ in $\mathfrak{D}_{f}^{\mathbb{R}}$ (resp. $\mathfrak{D}_{f}$ ), and every Borel subset $U$ of $W \cap \mathbb{R}$ (resp. $W$ ), we have

$$
\widetilde{\mu}\left(F_{f}(U)\right)=\exp \left(p m_{f}(W)\right) \int_{U}\left|D F_{f}\right|^{t} \mathrm{~d} \widetilde{\mu} .
$$


Note that in this case we have

$$
\begin{aligned}
\exp \left(-p m_{f}(W)\right) \inf _{z \in W}\left|D F_{f}(z)\right|^{-t} \leq \widetilde{\mu}(W) & \\
& \leq \exp \left(-p m_{f}(W)\right) \sup _{z \in W}\left|D F_{f}(z)\right|^{-t} .
\end{aligned}
$$

Proposition 3.4 ([CR17], Proposition 5.2). Let $n \geq 5$ be an integer, $f$ in $\mathcal{K}_{n}(\mathscr{F})$, and $t>0$ such that

$$
\mathscr{P}_{f}^{\mathbb{R}}\left(t, P_{f}^{\mathbb{R}}(t)\right)=0\left(\text { resp. } \mathscr{P}_{f}\left(t, P_{f}(t)\right)=0\right) .
$$

Then there is a $\left(t, P_{f}^{\mathbb{R}}(t)\right)$-conformal (resp. $\left(t, P_{f}(t)\right)$-conformal) probability measure $\widetilde{\mu}$ for $F_{f}$, and there is a probability measure $\widetilde{\rho}$ that is invariant by $F_{f}$, absolutely continuous with respect to $\widetilde{\mu}$, and whose density satisfies

$$
\Xi_{1}^{-t} \leq \frac{\mathrm{d} \widetilde{\rho}}{\mathrm{d} \widetilde{\mu}} \leq \Xi_{1}^{t}
$$

If in addition

$$
\begin{aligned}
& \sum_{W \in \mathcal{D}^{\mathbb{R}}} m_{f}(W) \cdot \exp \left(-m_{f}(W) P_{f}^{\mathbb{R}}(t)\right) \sup _{w \in W \cap \mathbb{R}}\left|D F_{f}(w)\right|^{-t} \\
&\left(\text { resp. } \sum_{W \in \mathfrak{D}} m_{f}(W) \cdot \exp \left(-m_{f}(W) P_{f}(t)\right) \sup _{w \in W}\left|D F_{f}(w)\right|^{-t}\right)
\end{aligned}
$$

is finite, then the measure

$$
\widehat{\rho}:=\sum_{W \in \mathfrak{D}^{\mathbb{R}}} \sum_{j=0}^{m_{f}(W)-1}\left(f^{j}\right)_{*}\left(\left.\widetilde{\rho}\right|_{W \cap \mathbb{R}}\right)\left(\operatorname{resp} . \sum_{W \in \mathfrak{D}} \sum_{j=0}^{m_{f}(W)-1}\left(f^{j}\right)_{*}\left(\left.\widetilde{\rho}_{t}\right|_{W}\right)\right)
$$

is finite and the probability measure proportional to $\widehat{\rho}$ is the unique equilibrium state of $\left.f\right|_{I(f)}$ (resp. $\left.\left.f\right|_{J(f)}\right)$ for the potential $-t \log |D f|$.

3.4. Statements of main results. In this subsection we state Theorems $A$ and $B$ These results are based on the notion of "admissible" family of quadratic-like maps introduced in [CR17, which we recall now. The proofs of both of these theorems are given in 4 .

Given a normalized quadratic-like map $f$, for each periodic point $p$ of $f$ of pe$\operatorname{riod} m$ in $\mathbb{N}$, put

$$
\chi_{f}(p):=\frac{1}{m} \log \left|D f^{m}(p)\right| .
$$

Definition 3.5 (Admissible family of quadratic-like maps). A uniform family of quadratic-like maps $\mathscr{F}$ is admissible, if for every sufficiently large integer $n \geq 6$ the following properties hold.

1. If we endow $\mathscr{F}$ with the topology of locally uniform convergence, then there is a continuous function $s_{n}: \mathcal{K}_{n} \rightarrow \mathcal{K}_{n}(\mathscr{F})$ such that $c \circ s_{n}$ is the identity.

2. For every $f$ in $s_{n}\left(\mathcal{K}_{n}\right)$, we have

$$
\chi_{f}(p(f))>\chi_{f}\left(p^{+}(f)\right) \text { and } \chi_{f}\left(p^{+}(f)\right)=\chi_{f}\left(p^{-}(f)\right) .
$$

Given a uniform family $\mathscr{F}$, for each integer $n \geq 5$, each $f$ in $\mathcal{K}_{n}(\mathscr{F})$ put

$$
\theta(f):=\left|\frac{D g_{f}(p(f))}{D g_{f}\left(p^{+}(f)\right)}\right|^{1 / 2} \text { and } t_{*}(f):=\frac{\log 2}{\log \theta(f)} .
$$


Note that $t_{*}(f)$ is a real analytic function of $f$.

Endow the set $\{+,-\}$ with the discrete topology, and $\{+,-\}^{\mathbb{N}}$ with the corresponding product topology.

Theorem A (Phase transitions). For every $R_{0}>0$ there is a constant $K_{0}>1$ such that if $\mathscr{F}_{0}$ is an admissible uniform family of quadratic-like maps with constants $K_{0}$ and $R_{0}$, then for every sufficiently large integer $n$ there is a continuous subfamily $\left(f_{\underline{\varsigma}}\right)_{\underline{\varsigma} \in\{+,-\}^{\mathbb{N}}}$ of $s_{n}\left(\mathcal{K}_{n}\right)$ such that the following properties hold.

For each $\underline{\varsigma}$ in $\{+,-\}^{\mathbb{N}}$ the map $f_{\underline{\varsigma}}$ is essentially topologically exact and we have $\chi_{\text {crit }}\left(f_{\underline{\varsigma}}\right)>0$. Moreover, for each positive $t<t_{*}\left(f_{\underline{\varsigma}}\right)$ there is a unique equilibrium state $\rho_{t}^{\mathbb{R}}(\underline{\varsigma})$ (resp. $\left.\rho_{t}(\underline{\varsigma})\right)$ of $\left.f_{\underline{\varsigma}}\right|_{I\left(f_{\underline{\varsigma}}\right)}\left(\right.$ resp. $\left.\left.f_{\underline{\varsigma}}\right|_{J\left(f_{\varsigma}\right)}\right)$ for the potential $-t \log \left|D f_{\underline{\varsigma}}\right|$.

Furthermore, there are positive numbers $q, \bar{\xi}, \Xi>2 \xi$, and $\Delta \geq 1$ such that for every $\subseteq$ in $\{+,-\}^{\mathbb{N}}$ the following property holds. Put

$$
t_{*}:=t_{*}\left(f_{\underline{\varsigma}}\right) \text { and } t_{0}:=\left(1-\frac{1}{400 q}\right) \cdot t_{*}
$$

and define the functions $\delta^{+}, \delta^{-}, P^{+}, P^{-}:\left(t_{0},+\infty\right) \rightarrow \mathbb{R}$, by

$$
\begin{aligned}
& \delta^{+}(t):= \begin{cases}\frac{2 \log 2}{3} \cdot 2^{-q\left(\left(\frac{t_{*}(\Xi-2 \xi)}{q\left(t_{*}-t\right)}\right)^{\frac{1}{2}}-1\right)^{3}} & \text { if } t \in\left(t_{0}, t_{*}\right) ; \\
0 & \text { if } t \geq t_{*} ;\end{cases} \\
& \delta^{-}(t):= \begin{cases}\frac{\log 2}{3} \cdot 2^{-q\left(\left(\frac{t_{*}(\Xi+2 \xi)}{q\left(t_{*}-t\right)}\right)^{\frac{1}{2}}+\Delta\right)^{3}} & \text { if } t \in\left(t_{0}, t_{*}\right) ; \\
0 & \text { if } t \geq t_{*} ;\end{cases} \\
& P^{+}(t):=-t \frac{\chi_{\text {crit }}\left(f_{\underline{\varsigma}}\right)}{2}+\delta^{+}(t) \text {, and } P^{-}(t):=-t \frac{\chi_{\text {crit }}\left(f_{\underline{\varsigma}}\right)}{2}+\delta^{-}(t) .
\end{aligned}
$$

Then for $t>t_{0}$ we have

$$
P^{-}(t) \leq P_{f_{\underline{\underline{s}}}}^{\mathbb{R}}(t) \leq P_{f_{\underline{\underline{\Sigma}}}}(t) \leq P^{+}(t),
$$

and for $t \geq t_{*}$ there is no equilibrium state of $\left.f_{\underline{\varsigma}}\right|_{I\left(f_{\underline{\varsigma}}\right)}$ (resp. $\left.\left.f_{\underline{\varsigma}}\right|_{J\left(f_{\varsigma}\right)}\right)$ for the potential $-t \log \left|D f_{\underline{\varsigma}}\right|$ and we have

$$
\mathscr{P}_{f_{\Sigma}}^{\mathbb{R}}\left(t,-t \frac{\chi_{\text {crit }}(f)}{2}\right) \leq \mathscr{P}_{f_{\underline{\Sigma}}}\left(t,-t \frac{\chi_{\text {crit }}(f)}{2}\right)<0 .
$$

Finally, for $t \geq t_{*}$ and for $p$ in $\mathbb{R}$ the following properties hold:

1. If $p \geq-t \chi_{\mathrm{crit}}(f) / 2$, then there is a unique $(t, p)$-conformal probability measure for $\left.f_{\underline{\varsigma}}\right|_{I\left(f_{\varsigma}\right)}$ (resp. $f_{\underline{\varsigma}}$ ) supported on $I\left(f_{\underline{\varsigma}}\right)$ (resp. $J\left(f_{\underline{\varsigma}}\right)$ ). Moreover, this measure is dissipative, purely atomic, and supported on the backward orbit of $z=0$.

2. If $p<-t \chi_{\text {crit }}(f) / 2$, then there is no $(t, p)$-conformal probability measure for $\left.f_{\underline{\varsigma}}\right|_{I\left(f_{\underline{\varsigma}}\right)}$ (resp. $f_{\underline{\varsigma}}$ ) supported on $I\left(f_{\underline{\varsigma}}\right)$ (resp. $J\left(f_{\underline{\varsigma}}\right)$ ).

For proving the High-Order Phase Transitions Theorem, observe that $t_{*}\left(f_{\underline{\varsigma}}\right)$ is continuous in the compact set $\{+,-\}^{\mathbb{N}}$.

Theorem B (Sensitive dependence). For every $R_{0}>0$ there is a constant $K_{0}^{\prime}>1$ such that if $\mathscr{F}_{0}$ is an admissible uniform family of quadratic-like maps with constants $K_{0}^{\prime}$ and $R_{0}$, then for every sufficiently large integer $n$ there is a continuous subfamily $\left(f_{\underline{\varsigma}}\right)_{\underline{\subseteq}} \in\{+,-\}^{\mathrm{N}}$ of $s_{n}\left(\mathcal{K}_{n}\right)$ satisfying the conclusions of Theorem $₫$ and such that the following property holds: 
There are constants $C>0$ and $\varkappa>0$, and a strictly increasing sequence of positive real numbers $\left(\tau_{m}\right)_{m \in \mathbb{N}}$ converging to 1 such that for every sequence $\underline{\varsigma}=$ $(\varsigma(m))_{m \in \mathbb{N}}$ in $\{+,-\}^{\mathbb{N}}$ the following properties hold. Put $t_{*}:=t_{*}\left(f_{\varsigma}\right)$, and let $m$ and $\widehat{m}$ be integers such that

$$
\widehat{m} \geq m \geq 1 \quad \text { and } \quad \varsigma(m)=\cdots=\varsigma(\widehat{m})
$$

and let $t$ be in $\left[t_{*} \tau_{m}, t_{*} \tau_{\widehat{m}}\right]$. Then the equilibrium state $\rho_{t}^{\mathbb{R}}(\underline{\varsigma})$ (resp. $\rho_{t}(\underline{\varsigma})$ ) of $\left.f_{\underline{\varsigma}}\right|_{I\left(f_{\underline{\varsigma}}\right)}$ (resp. $\left.\left.f_{\underline{\varsigma}}\right|_{J\left(f_{\underline{\varsigma}}\right)}\right)$ satisfies

$$
\begin{aligned}
\rho_{t}^{\mathbb{R}}(\underline{\varsigma}) & \left(B\left(\mathcal{O}^{\varsigma(m)}\left(f_{\underline{\varsigma}}\right), \exp \left(\frac{-\varkappa}{t_{*}-t}\right)\right)\right) \geq 1-C \exp \left(\frac{-\varkappa}{t_{*}-t}\right) \\
& \left(\operatorname{resp} . \rho_{t}(\underline{\varsigma})\left(B\left(\mathcal{O}^{\varsigma(m)}\left(f_{\underline{\varsigma}}\right), \exp \left(\frac{-\varkappa}{t_{*}-t}\right)\right)\right) \geq 1-C \exp \left(\frac{-\varkappa}{t_{*}-t}\right)\right) .
\end{aligned}
$$

Our estimates show that for every $\varsigma$ in $\{+,-\}^{\mathbb{N}}$ every accumulation measure of $\left(\rho_{t}^{\mathbb{R}}(\underline{\varsigma})\right)_{t>0}$ and of $\left(\rho_{t}(\underline{\varsigma})\right)_{t>0}$ is supported on $\mathcal{O}^{+}\left(f_{\underline{\varsigma}}\right) \cup \mathcal{O}^{-}\left(f_{\underline{\varsigma}}\right)$, see Remark 4.1 .

3.5. Proof of the Sensitive Dependence at Positive Temperature assuming Theorem B, Let $K_{0}^{\prime}$ be the constant given by Theorem $\mathrm{B}$ for $R_{0}=80$. Let $r_{\#}$ be the constant given by [CR17, Lemma 3.5] for this choice of $K_{0}^{\prime}$. Put $\Lambda_{0}=B\left(0, r_{\#}\right)$, and let $\mathscr{F}_{0}=\left(\widehat{f}_{\lambda}\right)_{\lambda \in \Lambda_{0}}$ be the family of quadratic-like maps constructed in [CR17, Lemma 3.5] for this choice of $K_{0}^{\prime}$. By [CR17, Lemma 3.5] and [CR17, (3.3)] the family $\mathscr{F}_{0}$ is uniform with constants $K_{0}^{\prime}$ and 80, and admissible. Fix a sufficiently large integer $n$ for which the conclusions of Theorem B are satisfied, and let $\left(f_{\underline{\varsigma}}\right)_{\underline{\varsigma} \in\{+,-\}^{\mathbb{N}}}$ and $\left(\tau_{m}\right)_{m \in \mathbb{N}}$ as in the statement of Theorem B Given $\underline{\varsigma}$ in $\{+,-\}^{\mathbb{N}}$, denote by $\lambda(\underline{\varsigma})$ the unique parameter in $\Lambda_{0}$ such that $\widehat{f}_{\lambda(\underline{\varsigma})}=f_{\underline{\varsigma}}$. By [CR17, Lemma 3.5], $\lambda(\underline{\varsigma})$ is real. We prove the Sensitive Dependence at Positive Temperature with $\Lambda=\left\{\lambda(\underline{\varsigma}) \mid \underline{\varsigma} \in\{+,-\}^{\mathbb{N}}\right\}$.

Let $\lambda_{0}$ in $\Lambda$ be given, and let $\left(\delta_{\ell}\right)_{\ell \in \mathbb{N}}$ be an increasing sequence of positive numbers converging to 1 as $\ell \rightarrow+\infty$. Let $\underline{\varsigma}_{0}$ in $\{+,-\}^{\mathbb{N}}$ be such that $\lambda_{0}:=\lambda\left(\underline{\varsigma}_{0}\right)$, and put $t_{*, 0}:=t_{*}\left(f_{\varsigma_{0}}\right)$. Replacing $\left(\delta_{\ell}\right)_{\ell \in \mathbb{N}}$ by a subsequence if necessary, assume that for every $\ell \in \mathbb{N}$ there is a unique $m \in \mathbb{N}$ such that

$$
\delta_{\ell} \in\left[\tau_{m}, \tau_{m+1}\right) \text { and } \delta_{\ell+1} \geq \tau_{m+2} .
$$

Put $m(\ell):=m$. We see that $m(\ell+1) \geq m(\ell)+2$, which implies

$$
\delta_{\ell} \in\left[\tau_{m(\ell)}, \tau_{m(\ell+1)-1}\right] .
$$

Let $M$ be a given positive integer. Let $\ell_{M}$ be the smallest $\ell$ such that $m(\ell) \geq M$. Let $\underline{\varsigma}=(\varsigma(m))_{m \in \mathbb{N}}$ be a sequence in $\{+,-\}^{\mathbb{N}}$ that agrees with $\underline{\varsigma}_{0}$ up to $m\left(\ell_{M}\right)$ and such that for every even (resp. odd) integer $\ell>\ell_{M}$ and every $m$ in $[m(\ell), m(\ell+$ $1)-1$ ] we have $\varsigma(m)=+($ resp. $\varsigma(m)=-)$.

Put $\lambda:=\lambda(\underline{\varsigma})$ and $t_{*}:=t_{*}\left(f_{\underline{\varsigma}}\right)$. Since the subfamily $\left(f_{\underline{\varsigma}}\right)_{\underline{\varsigma} \in\{+,-\}^{\mathrm{N}}}$ is continuous, taking $M$ sufficiently large we can ensure that $\lambda$ is arbitrarily close to $\lambda_{0}$ and that $t_{*}$ is arbitrarily close to $t_{*, 0}$.

By (3.8) we have that $t_{*}(\underline{\varsigma}) \cdot \delta_{\ell}$ is in $\left[t_{*}(\underline{\varsigma}) \cdot \tau_{m(\ell)}, t_{*}(\underline{\varsigma}) \cdot \tau_{m(\ell+1)-1}\right]$. Since by definition of $\underline{\varsigma}$ for each $\ell$ in $\mathbb{N}$ larger than $\ell_{M}$ we have

$$
\varsigma(m(\ell))=\cdots=\varsigma(m(\ell+1)-1),
$$


and since $\varsigma(m(\ell))$ alternates between + and - according to whether $\ell$ is even or odd, Theorem B implies the desired assertion for this choice of $\underline{\varsigma}$ and thus, for $\lambda$ and $t_{*}$.

\section{Proof of MAIn RESUlts}

In this section we prove Theorems $\mathrm{A}$ and $\mathrm{B}$ in $\$ 4.2$ and $\$ 4.3$ respectively. Indeed, for both theorems we use the same family of maps. More precisely, put $v:=\frac{1}{4} \log 2$, and let $R_{0}>0$ be given. Let $K_{1}, n_{1}$, and $\kappa_{1}$ be given by Proposition A.4 with $R^{\prime}=$ $R_{0}$. We prove that Theorems $\mathrm{A}$ and $\mathrm{B}$ hold with $K_{0}=K_{1}$. Let $\mathscr{F}_{0}$ be a uniform family of quadratic-like maps that admits $K_{0}$ and $R_{0}$ as uniformity constants, and that is admissible. By Proposition A.4 with $\mathscr{F}_{1}=\mathscr{F}_{0}$, for every $n \geq n_{1}$, every $f$ in $\mathcal{K}_{n}\left(\mathscr{F}_{0}\right)$ satisfies the Geometric Peierls Condition with constants $\kappa_{1}$ and $v$, and by A.1 we have

$$
\log \theta(f) \leq \frac{3}{8} v
$$

Taking $n_{1}$ larger if necessary, assume that for every $n \geq n_{1}$ there is a continuous function $s_{n}: \mathcal{K}_{n} \rightarrow \mathcal{K}_{n}\left(\mathscr{F}_{0}\right)$ such that $c \circ s_{n}$ is the identity, and that (3.6) holds for every $f$ in $s_{n}\left(\mathcal{K}_{n}\right)$.

Let $\Xi_{1}, \Xi_{2}, C_{0}, v_{0}$ and $\Xi_{3}$ be the constants given by Lemmas 3.3. A.1 A.2. and A.7 respectively, with $\mathscr{F}=\mathscr{F}_{0}$. Moreover, let $n_{2}$ and $C_{1}$ the constants given by Proposition A.5 with $\mathscr{F}=\mathscr{F}_{0}$ and $\kappa=\kappa_{1}$, let $n_{3}$ and $C_{2}$ be given by Proposition A.6 with $\mathscr{F}=\mathscr{F} 0$, and let $n_{\&} \geq \max \left\{n_{1}, n_{2}, n_{3}\right\}$ be sufficiently large so that

$$
\exp \left(n_{\&} v\right) \geq \Xi_{3}^{\frac{1}{2}} C_{1}\left(25+5 \cdot \Xi_{2}\right) .
$$

4.1. The subfamily. In this subsection we define the subfamily $\left(f_{\underline{\varsigma}}\right)_{\underline{\varsigma} \in\{+,-\}^{\mathbb{N}}}$, as in the statement of Theorems $\mathrm{A}$ and $\mathrm{B}$. Fix an integer $n \geq n_{\&}$, let $c_{\&}$ in $\mathcal{K}_{n}$ be such that $\iota\left(c_{\&}\right)$ is the constant sequence equal to 0 , and put $f_{\&}:=s_{n}\left(c_{\&}\right)$. By (3.6) we have $\theta\left(f_{\&}\right)>1$, so there is $r_{\&}>0$ such that for $c$ in $B\left(c_{\&}, r_{\&}\right) \cap$ $\mathcal{K}_{n}$ the number $\theta\left(s_{n}(c)\right)$ is defined, and depends continuously on $c$. Reducing $r_{\&}$ if necessary, assume that for all $c$ and $c^{\prime}$ in $B\left(c_{\&}, r_{\&}\right) \cap \mathcal{K}_{n}$ we have $\theta\left(s_{n}(c)\right) \leq$ $\theta\left(s_{n}\left(c^{\prime}\right)\right)^{2}$. By Proposition 2.4 it follows that there is an integer $q_{\&} \geq 0$ such that the set

$$
\left\{c \in \mathcal{K}_{n} \mid \text { for every } j \text { in }\left\{0, \ldots, q_{\&}\right\}, \iota(c)_{j}=0\right\}
$$

is a compact set contained in $B\left(c_{\&}, r_{\&}\right)$. Put

$$
\begin{gathered}
t_{*, \sup }:=\sup _{c \in \mathcal{K}_{n} \cap B\left(c_{\&}, r_{\&}\right)} \frac{\log 2}{\log \theta\left(s_{n}(c)\right)}, t_{*, \text { inf }}:=\inf _{c \in \mathcal{K}_{n} \cap B\left(c_{\&}, r_{\&}\right)} \frac{\log 2}{\log \theta\left(s_{n}(c)\right)}, \\
\eta_{0}:=\sup _{f \in s_{n}\left(\mathcal{K}_{n} \cap B\left(c_{\&}, r_{\&}\right)\right)} \frac{\exp \left(\chi_{f}(\beta(f))\right)}{\exp \left(\chi_{\text {crit }}(f)\right)},
\end{gathered}
$$

and fix $\Omega>0$ sufficiently large such that

$$
2^{2 \Omega-3} \geq 2^{n}\left(\Xi_{3}^{\frac{1}{2}} C_{1} \eta_{0}^{\frac{n}{2}}\right)^{t_{*, \text { sup }}} .
$$

On the other hand, by (3.6) for each $c$ in $\mathcal{K}_{n}$ the number

$$
\xi\left(s_{n}(c)\right)=\frac{\log \Xi_{2}}{2 \log \theta\left(s_{n}(c)\right)},
$$


defined as in $\$$ A.4 depends continuously on $c$ in $\mathcal{K}_{n}$, so

$$
\xi:=\sup _{c \in \mathcal{K}_{n} \cap B\left(c_{\&}, r_{\&}\right)} \xi\left(s_{n}(c)\right)<+\infty .
$$

Note that by our choice of $r_{\&}$ for every $c \in \mathcal{K}_{n} \cap B\left(c_{\&}, r_{\&}\right)$ we have

$$
\xi \leq 2 \xi\left(s_{n}(c)\right) .
$$

Put $\Xi:=\lceil 2 \xi\rceil+1$ as in $\$ \underline{\mathrm{B} .1}$, and let $q \geq q_{\&}$ be a sufficiently large integer satisfying the conditions in $\$ \mathrm{~B} .1$ and such that in addition $q+\Xi$ is even. For each real number $s \geq 0$, let $I_{s}$ and $J_{s}$ be the intervals defined in $\$$ B.1 for these choices of $\Xi$ and $q$. By definition, as $s$ varies in $\mathbb{N}_{0}$ these intervals form a partition of $[1,+\infty)$. Moreover, for each integer $s$ in $\mathbb{N}_{0}$, the end points of $I_{s}$ and $J_{s}$ are even.

Endow the set $\left\{0,1^{+}, 1^{-}\right\}$with the discrete topology, and $\left\{0,1^{+}, 1^{-}\right\}^{\mathbb{N}_{0}}$ with the corresponding product topology. Moreover, endow the subset $\widehat{\Sigma}$ of $\left\{0,1^{+}, 1^{-}\right\}^{\mathbb{N}_{0}}$, defined in A.4 with the induced topology.

As in $\leqq .2$, for every $\tau \in(0,1)$, put

$$
s^{+}(\tau):=\left(\frac{\Xi-2 \xi}{q(1-\tau)}\right)^{\frac{1}{2}} \text { and } s^{-}(\tau):=\left(\frac{\Xi+2 \xi}{q(1-\tau)}\right)^{\frac{1}{2}} .
$$

Let $\left(\tau_{m}\right)_{m \in \mathbb{N}_{0}}$ be an increasing sequence in $[1 / 2,1)$ converging to 1 satisfying the following properties:

(4.7) For every $m \in \mathbb{N}$, we have $s^{+}\left(\tau_{m}\right) \in \mathbb{N}$, and $s^{-}\left(\tau_{m-1}\right)+\Omega+6<s^{+}\left(\tau_{m}\right)$.

Notice that the inequality $\Xi-2 \xi \geq 1$ implies that for every $\tau \geq 1-\frac{1}{400 q}$ we have

$$
s^{+}(\tau) \geq 20 \text {. }
$$

Put $\ell(0)=0$ and for every $m$ in $\mathbb{N}$ put $\ell(m):=s^{+}\left(\tau_{m}\right)-5$. Given $\underline{\varsigma}$ in $\{+,-\}^{\mathbb{N}}$, let $\underline{\widehat{x}}(\underline{\varsigma})$ be the sequence in $\left\{0,1^{+}, 1^{-}\right\}^{\mathbb{N}_{0}}$ defined by

$$
\underline{\widehat{x}}(\underline{\varsigma})_{j}:= \begin{cases}0 & \text { if for some } s \text { in } \mathbb{N}_{0} \text { we have } j+1 \in I_{s} ; \\ 1^{+} & \text {if } j+1 \in \bigcup_{k \in[\ell(0), \ell(1))} J_{k} ; \\ 1^{\varsigma(m)} & \text { for } j+1 \in \bigcup_{k \in[\ell(m), \ell(m+1))} J_{k} .\end{cases}
$$

Note that by definition $\underline{\widehat{x}}(\underline{\varsigma})$ is in $\widehat{\Sigma}$ and that the first $q$ entries of this sequence are equal to 0 . Moreover, the map $\underline{\widehat{x}}:\{+,-\}^{\mathbb{N}} \rightarrow \widehat{\Sigma}$ so defined is continuous. Finally, note that the length of each maximal block of $1^{-}$'s in $\underline{\widehat{x}}(\underline{\varsigma})$ is even.

Define the family of itineraries $(\iota(\underline{\varsigma}))_{\underline{\varsigma} \in\{+,-\}^{\mathbb{N}}}$ in $\{0,1\}^{\mathbb{N}_{0}}$, by

$$
\iota(\underline{\varsigma})_{j}= \begin{cases}0 & \text { if } \underline{\widehat{x}}(\underline{\varsigma})_{j}=0 ; \\ 1 & \text { if } \underline{\widehat{x}}(\underline{\varsigma})_{j}=1^{+} ; \\ 0 & \text { if } \underline{\widehat{x}}(\underline{\varsigma})_{j}=1^{-} \text {and } j \text { is even; } \\ 1 & \text { if } \underline{\widehat{x}}(\underline{\varsigma})_{j}=1^{-} \text {and } j \text { is odd. }\end{cases}
$$

Note that $\iota(\underline{\varsigma})$ is compatible with $\underline{\widehat{x}}(\underline{\varsigma})$ in the sense of $\$$ A.4 and that the first $q$ entries of this sequence are equal to 0 . Moreover, $\iota(\underline{\varsigma})$ depends continuously on $\underline{\varsigma}$ in $\{+,-\}^{\mathbb{N}}$. 
Given $\underline{\varsigma}$ in $\{+,-\}^{\mathbb{N}}$, let $c(\underline{\varsigma})$ in $\mathcal{K}_{n}$ be the unique parameter such that $\iota\left(f_{c(\varsigma)}\right)=$ $\iota(\underline{\varsigma})$ (Proposition 2.4), and put $f_{\underline{\varsigma}}:=s_{n}(c(\underline{\varsigma}))$. Note that the function $\underline{\varsigma} \mapsto f_{\varsigma}$ so defined is continuous. On the other hand, since for each $\subseteq$ in $\{+,-\}^{\mathbb{N}}$ the first $q \geq q_{\&}$ entries of $\iota\left(f_{\underline{\varsigma}}\right)=\iota(\underline{\varsigma})$ are equal to 0 , the parameter $c(\underline{\varsigma})$ is in $B\left(\lambda_{\&}, r_{\&}\right)$. So, for every $\underline{\varsigma}$ and $\underline{\varsigma}^{\prime}$ in $\{+,-\}^{\mathbb{N}}$ we have

$$
\theta\left(f_{\underline{\varsigma}}\right) \leq \theta\left(f_{\underline{\varsigma}^{\prime}}\right)^{2} .
$$

This finishes the definition of the continuous subfamily $\left(f_{\underline{\varsigma}}\right)_{\underline{\varsigma} \in\{+,-\}^{\mathbb{N}}}$ of $s_{n}\left(\mathcal{K}_{n}\right)$.

4.2. Proof of Theorem $\mathbf{A}$. The purpose of this subsection is to finish the proof of Theorem $\underline{\mathrm{A}}$. That for each $\underline{\varsigma}$ in $\{+,-\}^{\mathbb{N}}$ the map $\left.f_{\underline{\varsigma}}\right|_{I\left(f_{\underline{\varsigma}}\right)}$ is essentially topologically exact follows from the fact that this map is not renormalizable, see [CRL13, §3] for details. To prove that for each $\underline{\varsigma}$ in $\{+,-\}^{\mathbb{N}}$ there is a unique equilibrium state for every positive $t<t_{*}\left(f_{\underline{\varsigma}}\right)$ we just need to show that $P_{f_{\varsigma}}^{\mathbb{R}}(t)>-t \cdot \chi_{\text {crit }}\left(f_{\underline{\varsigma}}\right) / 2$. This follows from the estimates for the pressure function given below. These estimates will be also used in the proof of Theorem B in the next section, and they depend only on Lemma B.2.

Let $N: \mathbb{N}_{0} \rightarrow \mathbb{N}_{0}$ and $B: \mathbb{N}_{0} \rightarrow \mathbb{N}_{0}$ be the functions defined in $\$$ B.1 for our choices of $\Xi$ and $q$. Clearly, $N(k) / k \rightarrow 0$ as $k \rightarrow+\infty$, and for each $\varsigma$ in $\{+,-\}^{\mathbb{N}}$, these functions coincide with those defined in $\$$ A.4 with $\left(\widehat{x}_{j}\right)_{j \in \mathbb{N}_{0}}=\underline{\widehat{x}}(\underline{\varsigma})$. It follows that for each integer $k \geq 0$, the 2 variables functions $\pi_{f_{\underline{s}}, k}^{ \pm}$defined in $\$$ A.4 for our choice of $\xi$ in 4.1 are independent of $\underline{\text { }}$; we denote them by $\pi_{k}^{ \pm}$. Note that the 2 variables series defined in $\$ \mathrm{~B} .1$. $\Pi^{ \pm}$, and for each integer $s \geq 0$, the series $I_{s}^{ \pm}$, and $J_{s}^{ \pm}$, satisfy

$$
\Pi^{ \pm}=\sum_{k=0}^{+\infty} \pi_{k}^{ \pm}, I_{s}^{ \pm}=\sum_{k \in I_{s}} \pi_{k}^{ \pm}, \text {and } J_{s}^{ \pm}=\sum_{k \in J_{s}} \pi_{k}^{ \pm} .
$$

Finally, for each real number $s \geq 0$ put $\lambda(s)=\left|J_{s}\right|^{-1}$, as in $\$$ B.1

Fix $\underline{\varsigma}$ in $\{+,-\}^{\mathbb{N}}$ and put

$$
\begin{gathered}
f:=f_{\underline{\varsigma}}, p^{+}:=p^{+}(\underline{\varsigma}), p^{-}:=p^{-}(\underline{\varsigma}), \\
P^{\mathbb{R}}:=P_{f_{\underline{\varsigma}}}^{\mathbb{R}}, \mathscr{P}^{\mathbb{R}}:=\mathscr{P}_{f_{\varsigma}}^{\mathbb{R}}, P:=P_{f_{\underline{\varsigma}}}, \mathscr{P}:=\mathscr{P}_{f_{\underline{\varsigma}}}, \\
t_{*}:=t_{*}\left(f_{\underline{\varsigma}}\right), \text { and } t_{0}:=\left(1-\frac{1}{400 q}\right) \cdot t_{*} .
\end{gathered}
$$

Now we prove the estimates for the pressure functions for every $t$ in $\left(t_{0}, t_{*}\right)$. Fix $t$ in $\left(t_{0}, t_{*}\right)$ and put

$$
\tau:=\frac{1}{t_{*}} t=\frac{\log \theta(f)}{\log 2} t .
$$

By (4.8), we have $s^{+}(\tau)-2 \geq 18$. Put

$\widehat{P}^{+}:=-t \frac{\chi_{\text {crit }}(f)}{2}+\frac{\log 2}{3} \lambda\left(s^{+}(\tau)-2\right), \quad$ and $\quad \widehat{P}^{-}:=-t \frac{\chi_{\text {crit }}(f)}{2}+\frac{\log 2}{3} \lambda\left(s^{-}(\tau)+\Omega\right)$.

By (4.1) we have that

$$
t_{0} \geq 8=\frac{2 \log 2}{v}
$$


First we prove the lower bound for the real pressure function. By (3.6), (B.5), Lemma A.7 with $\underline{\widehat{x}}=\underline{\widehat{x}}(\underline{\varsigma})$ and $\delta=\frac{\log 2}{3} \lambda\left(s^{-}(\tau)+\Omega\right) \leq \log 2$, Lemma B.2. the definition of $\eta_{0}$, and (4.3) we have

$$
\sum_{k=0}^{+\infty} \exp \left(-(n+3 k) \widehat{P}^{-}\right)\left|D f^{n+3 k}(f(0))\right|^{-\frac{t}{2}}
$$

$$
\begin{aligned}
& \geq \Xi_{3}^{-\frac{t}{2}} \exp \left(-n \frac{\log 2}{3} \lambda\left(s^{-}(\tau)+\Omega\right)\right)\left(\frac{\exp \left(\chi_{\text {crit }}(f)\right)}{|D f(\beta(f))|}\right)^{\frac{t}{2} n} \Pi^{-}\left(\tau, \lambda\left(s^{-}(\tau)+\Omega\right)\right) . \\
& \geq 2^{-n}\left(\Delta^{\frac{1}{2}} \eta_{0}^{\frac{n}{2}}\right)^{-t} 2^{2 \Omega-3} \\
& \geq C_{1}^{t} .
\end{aligned}
$$

By (A.1) we have $2 v \leq \chi_{\text {crit }}(f)$. Thus, using that $\lambda(s)$ is strictly decreasing, by part 1 of Lemma B.1, and (B.5), we deduce

$$
\widehat{P}^{-}<\widehat{P}^{+}<0 .
$$

Together with (4.10) and using part 1 of Proposition A.5, we get that

$$
P(t) \geq P^{\mathbb{R}}(t) \geq \widehat{P}^{-} \quad \text { and } \quad \mathscr{P}\left(t, \widehat{P}^{-}\right) \geq \mathscr{P}^{\mathbb{R}}\left(t, \widehat{P}^{-}\right)>0 .
$$

Putting $\Delta:=\Omega+1$, we get $P^{\mathbb{R}}(t) \geq \widehat{P}^{-} \geq P^{-}(t)$, where $P^{-}(t)$ as in the statement of Theorem A.

Now we prove the upper bound for the complex pressure function. Notice that by (4.4) and (4.9) we have

$$
2^{\tau \xi}=\theta(f)^{t \xi} \leq \theta(f)^{2 t \xi(f)}=\Xi_{2}^{t} .
$$

Combined with (3.6), Lemma A.7 with $\underline{\widehat{x}}=\underline{\widehat{x}}(\underline{\varsigma})$ and $\delta=\frac{\log 2}{3} \lambda\left(s^{+}(\tau)-2\right)$, Lemma B.2, and (4.2), we obtain that

$$
\begin{aligned}
\sum_{k=0}^{+\infty} \exp \left(-(n+3 k) \widehat{P}^{+}\right) \mid D f^{n+3 k} & \left.(f(0))\right|^{-\frac{t}{2}} \\
& \leq \Xi_{3}^{\frac{1}{2}}\left(\frac{\exp \left(\chi_{\text {crit }}(f)\right)}{|D f(\beta(f))|}\right)^{\frac{t}{2} n} \Pi^{+}\left(\tau, \lambda\left(s^{+}(\tau)-2\right)\right) . \\
& \leq\left(\Xi_{3}^{\frac{1}{2}} \exp (-n v)\right)^{t}\left(25+5 \cdot 2^{\tau \xi}\right) \\
& \leq\left(\Xi_{3}^{\frac{1}{2}} \exp (-n v)\left(25+5 \cdot \Xi_{2}\right)\right)^{t} \\
& \leq C_{1}^{-t} .
\end{aligned}
$$

Together with part 2 of Proposition A.5, this implies

$$
P^{\mathbb{R}}(t) \leq P(t) \leq \widehat{P}^{+} \quad \text { and } \quad \mathscr{P}^{\mathbb{R}}\left(t, \widehat{P}^{+}\right) \leq \mathscr{P}\left(t, \widehat{P}^{+}\right)<0 .
$$

To conclude we observe that by part 2 of Lemma B.1 we have that $\widehat{P}^{+} \leq P^{+}(t)$, where $P^{+}(t)$ is as in the statement of Theorem $\mathrm{A}$ This finish the prove of the estimates for the pressure functions for $t$ in $\left(t_{0}, t_{*}\right)$.

Now we prove the existence and uniqueness of equilibrium states for every $t$ in $\left(0, t_{*}\right)$. Put

$$
\chi_{\mathrm{inf}}^{\mathbb{R}}(f):=\inf \left\{\int \log |D f| \mathrm{d} \mu \mid \mu \in \mathscr{M}_{f}^{\mathbb{R}}\right\},
$$


and

$$
\chi_{\inf }(f):=\inf \left\{\int \log |D f| \mathrm{d} \mu \mid \mu \in \mathscr{M}_{f}\right\} .
$$

Combining (4.12) and (4.13), we deduce that

$$
P\left(t_{*}\right)=P^{\mathbb{R}}\left(t_{*}\right)=\lim _{t \nearrow t_{*}} P^{\mathbb{R}}(t)=-t_{*} \frac{\chi_{\text {crit }}(f)}{2} .
$$

Using that for every $t>0$ we have $P^{\mathbb{R}}(t) \geq-t \chi_{\text {inf }}^{\mathbb{R}}(f)$, we deduce that $\chi_{\text {inf }}^{\mathbb{R}}(f) \geq$ $\chi_{\text {crit }}(f) / 2$, and by [CR17, Proposition 5.6] we get $\chi_{\text {inf }}^{\mathbb{R}}(f)=\chi_{\text {crit }}(f) / 2$. Similarly, $\chi_{\mathrm{inf}}(f)=\chi_{\mathrm{crit}}(f) / 2$. Using (4.12) again, we conclude that for every $t$ in $\left(0, t_{*}\right)$ we have

$$
P^{\mathbb{R}}(t)>-t \chi_{\mathrm{inf}}^{\mathbb{R}}(f) \text { and } P(t)>-t \chi_{\mathrm{inf}}(f) .
$$

The existence and uniqueness of equilibrium states follows from [PR14 in the real case and from [PRL11] in the complex case.

For the estimates for the pressure functions for $t \geq t_{*}$, observe that

$$
P\left(t_{*}\right)=P^{\mathbb{R}}\left(t_{*}\right)=-t_{*} \chi_{\text {inf }}^{\mathbb{R}}(f)
$$

and the definition of the pressure function imply that for every $t$ in $\left[t_{*},+\infty\right)$ we have

$$
P(t)=P^{\mathbb{R}}(t)=-t \frac{\chi_{\text {crit }}(f)}{2} .
$$

Finally, we prove for $t \geq t_{*}$ the assertion about the $(t, p)$-conformal measures and the non-existence of equilibrium states. Using (3.6), Lemma A.7 with $\underline{\widehat{x}}=\underline{\widehat{x}}(\underline{\varsigma})$ and $\delta=0$, part 1 of Sublemma B.3, and (4.2), this implies that for every $t$ in $\left[t_{*},+\infty\right)$,

$$
\begin{aligned}
\sum_{k=0}^{+\infty} \exp \left(-(n+3 k)\left(-t \chi_{\mathrm{crit}}(f) / 2\right)\right) \mid D f^{n+3 k} & \left.(f(0))\right|^{-\frac{t}{2}} \\
& \left.\leq \Xi_{3}^{\frac{1}{2}}\left(\frac{\exp \left(\chi_{\mathrm{crit}}(f)\right)}{|D f(\beta(f))|}\right)^{\frac{t}{2} n} \Pi^{+}(\tau, 0)\right) . \\
& \leq\left(\Xi_{3}^{\frac{1}{2}} \exp (-n v)\right)^{t} 2\left(2^{\tau \xi}+1\right) \\
& \leq\left(\Xi_{3}^{\frac{1}{2}} \exp (-n v)\left(2+2 \cdot \Xi_{2}\right)\right)^{t} \\
& \leq C_{1}^{-t} .
\end{aligned}
$$

Together with part 2 of Proposition A.5, this implies that for every $t$ in $\left[t_{*},+\infty\right)$,

$$
\mathscr{P}^{\mathbb{R}}\left(t,-t \frac{\chi_{\text {crit }}(f)}{2}\right) \leq \mathscr{P}\left(t,-t \frac{\chi_{\text {crit }}(f)}{2}\right)<0 .
$$

Thus, for every $p \geq-\chi_{\text {crit }}(f) / 2$ we get that

$$
\mathscr{P}^{\mathbb{R}}(t, p) \leq \mathscr{P}(t, p) \leq \mathscr{P}\left(t,-t \frac{\chi_{\text {crit }}(f)}{2}\right)<0 .
$$

Hence, the assertion about $(t, p)$-conformal measures follows from (4.14), Proposition 3.2 and [CR17, Lemma 5.5].

To prove the assertions about equilibrium states, let $t \geq t_{*}$ be given and suppose by contradiction there is an equilibrium state $\rho$ of $\left.f\right|_{I(f)}$ (resp. $\left.f\right|_{J(f)}$ ) for 
the potential $-t \log |D f|$. Since $f$ satisfies the Collet-Eckmann condition, it follows that the Lyapunov exponent of $\rho$ is strictly positive, see [NS98, Theorem A] or [Riv12, Main Theorem] for the real case and [PRLS03, Main Theorem] for the complex case. Then [Dob15, Theorem 6] in the real case and [Dob12, Theorem 8] in the complex case imply that $\rho$ is absolutely continuous with respect to the $\left(t,-t \chi_{\text {crit }}(f) / 2\right)$-conformal measure for $\left.f\right|_{I(f)}$ (resp. $f$ ) that is supported on $I(f)$ (resp. $J(f)$ ). This implies in particular that $\rho$ is supported on the backward orbit of $z=0$ and hence that $\rho$ charges $z=0$. This is impossible because this point is not periodic. This contradiction shows that there is no equilibrium state of $\left.f\right|_{I(f)}$ (resp. $\left.f_{J(f)}\right)$ for the potential $-t \log |D f|$ and completes the proof Theorem $\mathrm{A}$.

4.3. Proof of Theorem B. In this subsection we complete the proof of Theorem $\mathrm{B}$. We give the proof in the complex setting; except for the obvious notational changes, it applies to the real case without modifications. The proof is based on the fact that for each $\underline{\varsigma}$ in $\{+,-\}^{\mathbb{N}}$ the equilibrium state $\rho_{t}(\underline{\varsigma})$ is absolutely continuous with respect to some $\left(t, P_{f_{\Sigma}}(t)\right)$-conformal measure with a density bounded from above and from below (see Proposition 3.4). Then, we can estimate the measure via the derivative of the orbit of the critical point. We keep the same notation as in the previous subsections.

Fix $t$ in $\left(t_{0}, t_{*}\right)$ and let $m_{0}$ be in $\mathbb{N}$ such that $t$ is in $\left[t_{*} \tau_{m_{0}-1}, t_{*} \tau_{m_{0}}\right]$. Observe that $\tau_{m_{0}-1} \leq \tau \leq \tau_{m_{0}}$. On the other hand, note that by (4.12) and (4.13) there is $s^{\mathbb{C}}$ in $\left[s^{+}(\tau)-2, s^{-}(\tau)+\Omega\right]$ such that

$$
P(t)=-t \frac{\chi_{\mathrm{crit}}(f)}{2}+\frac{\log 2}{3} \lambda\left(s^{\mathbb{C}}\right) .
$$

We start by proving that the hypotheses of Proposition 3.4 are satisfied for this value of $t$. By (3.6), Lemma A.7 with $\delta=\frac{\log 2}{3} \lambda\left(s^{-}(\tau)+\Omega\right)$, and part 1 of Lemma B.4, we have

$$
\sum_{k=0}^{+\infty} k \cdot \exp \left(-(n+3 k) \widehat{P}^{-}\right)\left|D f^{n+3 k}(f(0))\right|^{-\frac{t}{2}}<+\infty .
$$

In particular, this implies that the sum in (4.11) is finite, so by part 1 of Proposition A.5 we have

$$
\mathscr{P}\left(t, \widehat{P}^{-}\right)<+\infty \text {. }
$$

This implies that $\mathscr{P}(t, \cdot)$ is continuous and strictly decreasing on $\left[\widehat{P}^{-},+\infty\right)$, so by (4.12), (4.13), and [CR17, Proposition II] we have (3.3). Combining (4.16) and part 3 of Proposition A.5, we obtain (3.5). In view of (4.12), this implies the same sum in (3.5) with $\widehat{P}^{-}$replaced by $P(t)$, is finite. This complete the proof that the hypotheses of Proposition 3.4 are satisfied.

Let $\widetilde{\rho}$ and $\widehat{\rho}$ be the measures given by Proposition 3.4 Put $\mathfrak{D}:=\mathfrak{D}_{f}, F:=F_{f}$ and for every integer $k \geq 0$ put $\mathfrak{D}_{k}:=\mathfrak{D}_{f, k}$. For each integer $s \geq 0$ let $a_{s}$ and $b_{s}$ be the left and right endpoint of $I_{s}$, respectively as in B.1. Thus $a_{0}=1$ and for every integer $s \geq 0$ we have

$$
I_{s}=\left[a_{s}, b_{s}\right) \text { and } J_{s}=\left[b_{s}, a_{s+1}\right) .
$$

Note that by part 2 of Lemma B.1 and the hypothesis $q \geq 100(\Xi+1)$ in $\$$ B.1 we have $a_{s+1}-b_{s}=\left|J_{s}\right|>(s+1)^{2}$. 
Put $s_{0}:=\left\lceil s^{\mathbb{C}}\right\rceil$ and for each integer $\varsigma$ in $\left[s_{0}-3, s_{0}\right]$ put

$$
\widehat{\rho}_{\varsigma}^{\prime}:=\sum_{k=b_{\varsigma}+\varsigma^{2}}^{a_{\varsigma}-1} \sum_{j=n+3 b_{\varsigma}-2}^{n+3\left(k+1-\varsigma^{2}\right)} \sum_{W \in \mathfrak{D}_{k}}\left(f^{j}\right)_{*}\left(\left.\widetilde{\rho}_{t}\right|_{W}\right),
$$

and put

$$
\widehat{\rho}^{\prime \prime}:=\sum_{k \in J_{s_{0}}} \sum_{j=n+3 b_{s_{0}-1}-2}^{n+3\left(a_{s_{0}}+1-s_{0}^{2}\right)} \sum_{W \in \mathfrak{D}_{k}}\left(f^{j}\right)_{*}\left(\left.\widetilde{\rho}_{t}\right|_{W}\right)
$$

Finally, put

$$
\widehat{\rho}^{\prime}:=\left(\sum_{\varsigma=s_{0}-3}^{s_{0}} \hat{\rho}_{\varsigma}^{\prime}\right)+\widehat{\rho}^{\prime \prime}
$$

In part 1 we estimate the total mass of $\widehat{\rho}^{\prime}$ from below, and in part 2 we show that the total mass of $\widehat{\rho}_{t}-\widehat{\rho}^{\prime}$ is small in comparison to that of $\widehat{\rho}^{\prime}$. In part 3 we complete the proof of Theorem B by showing that the measure $\hat{\rho}^{\prime}$ is supported on a small neighborhood of the orbit of $p^{+}$if $\varsigma(m)=+$, and on a small neighborhood of the orbit of $p^{-}$if $\varsigma(m)=-$.

Similarly to the previous subsection note that the 2 variables series defined in $₫$ B.3. $\widetilde{\Pi}^{ \pm}$, and for each integer $s \geq 0$, the series $\widetilde{I}_{s}^{+}, \widetilde{J}_{s}^{+}$, and $\widehat{J}_{s}^{ \pm}$, satisfy

$$
\widetilde{\Pi}^{+}=\sum_{k=0}^{+\infty} k \cdot \pi_{k}^{+}, \widetilde{I}_{s}^{+}=\sum_{k \in I_{s}} k \cdot \pi_{k}^{+}, \widetilde{J}_{s}^{+}=\sum_{k \in J_{s}} k \cdot \pi_{k}^{+} \text {and } \widehat{J}_{s}^{ \pm}=\sum_{k=b_{s}+s^{2}}^{a_{s+1}-1}\left(k+1-b_{s}-s^{2}\right) \pi_{k}^{ \pm} .
$$

1. To estimate the total mass of $\widehat{\rho}^{\prime}$ from below put $\Upsilon_{1}:=\Xi_{1} C_{2} \Xi_{3}^{\frac{1}{2}} \eta_{0}^{\frac{n}{2}} 2^{n}$. Let $\varsigma$ be an integer in $\left[s_{0}-3, s_{0}\right]$. By part 1 of Proposition A.6. Lemma A.7 with $\delta=$ $\frac{\log 2}{3} \lambda\left(s^{\mathbb{C}}\right) \leq \log 2$, (3.2), (3.4), and (4.15), we have

$$
\begin{aligned}
\left|\hat{\rho}_{\varsigma}\right| & =\sum_{k=b_{\varsigma}+\varsigma^{2}}^{a_{\varsigma+1}-1} 3\left(k+1-b_{\varsigma}-\varsigma^{2}\right) \sum_{W \in \mathfrak{D}_{k}} \widetilde{\rho}_{t}(W) \\
& \geq\left(\Xi_{1} C_{2}\right)^{-t} \sum_{k=b_{\varsigma}+\varsigma^{2}}^{a_{\varsigma+1}-1} 3\left(k+1-b_{\varsigma}-\varsigma^{2}\right) \exp (-(n+3 k) P(t))\left|D f^{n+3 k}(f(0))\right|^{-t / 2} \\
& \geq\left(\Xi_{1} C_{2} \Xi_{3}^{\frac{1}{2}}\left(\frac{\left|D f\left(\beta\left(f_{\varsigma}\right)\right)\right|}{\exp \left(\chi_{\mathrm{crit}}(f)\right)}\right)^{\frac{n}{2}}\right)^{-t} 2^{-n} \sum_{k=b_{\varsigma}+\varsigma^{2}}^{a_{\varsigma+1}-1} 3\left(k+1-b_{\varsigma}-\varsigma^{2}\right) \pi_{k}^{-}\left(\tau, \lambda\left(s^{\mathbb{C}}\right)\right) \\
& \geq 3 \Upsilon_{1}^{-t} \widehat{J}_{\varsigma}^{-}\left(\tau, \lambda\left(s^{\mathbb{C}}\right)\right) .
\end{aligned}
$$

This implies

$$
\left|\widehat{\rho}^{\prime}\right| \geq 3 \Upsilon_{1}^{-t} \sum_{\varsigma=s_{0}-3}^{s_{0}} \widehat{J}_{\varsigma}^{-}\left(\tau, \lambda\left(s^{\mathbb{C}}\right)\right)
$$

2. For $\varsigma$ in $\left[s_{0}-3, s_{0}\right]$, put

$$
H_{\varsigma}:=\left\{k \in \mathbb{N}_{0} \mid b_{\varsigma}+\varsigma^{2} \leq k \leq a_{\varsigma+1}-1\right\} .
$$


By part 2 and the last inequality of Proposition A.6, (3.2), (3.4), and (4.15), we have

$$
\begin{aligned}
& |\widehat{\rho}-\widehat{\rho}|=\sum_{\substack{k \in \mathbb{N}_{0} \\
k \notin \bigcup_{\varsigma}^{s}=\tau_{0}-3}} \sum_{W \in \mathfrak{D}_{k}} m_{f}(W) \widetilde{\rho}(W) \\
& +\sum_{\varsigma=\tau_{0}-3}^{s_{0}-1} \sum_{k \in H_{\varsigma}} \sum_{W \in \mathfrak{D}_{k}}\left(m_{f}(W)-3\left(k+2-b_{\varsigma}-\varsigma^{2}\right)\right) \widetilde{\rho}(W) \\
& \left.+\sum_{k \in H_{s_{0}}} \sum_{W \in \mathfrak{D}_{k}}\left(m_{f}(W)-3\left(k+4-b_{s_{0}}-2 s_{0}^{2}+\left|J_{s_{0}-1}\right|\right)\right)\right) \tilde{\rho}(W) \\
& \leq\left(\Xi_{1} C_{2}\right)^{t}\left[\sum_{\substack{k \in \mathbb{N}_{0} \\
k \notin \bigcup_{\varsigma}^{s_{0}=\tau_{0}-3} H_{\varsigma}}}(n+3 k+1) \exp (-(n+3 k) P(t))\left|D f^{n+3 k}(f(0))\right|^{-\frac{t}{2}}\right. \\
& +\sum_{\varsigma=\tau_{0}-3}^{s_{0}-1} \sum_{k \in H_{\varsigma}}\left(n+3\left(b_{\varsigma}+\varsigma^{2}\right)\right) \exp (-(n+3 k) P(t))\left|D f^{n+3 k}(f(0))\right|^{-\frac{t}{2}} \\
& \left.+\sum_{k \in H_{s_{0}}}\left(n+3\left(b_{s_{0}}+2 s_{0}^{2}-\left|J_{s_{0}-1}\right|\right)\right) \exp (-(n+3 k) P(t))\left|D f^{n+3 k}(f(0))\right|^{-\frac{t}{2}}\right] \text {. }
\end{aligned}
$$

Thus, if we put $\Upsilon_{2}:=\Xi_{1} C_{2} \Xi_{3}^{\frac{1}{2}} \exp (-n v)$, then by (3.6), Lemma A.7 with $\delta=$ $\frac{\log 2}{3} \lambda\left(s^{\mathbb{C}}\right),(3.2)$, and part 2 of Lemma B.4,

$$
\begin{aligned}
\left|\widehat{\rho}-\widehat{\rho}^{\prime}\right| & \leq(n+4) \Upsilon_{2}^{t}\left[\widetilde{\Pi}^{+}\left(\tau, \lambda\left(s^{\mathbb{C}}\right)\right)-\sum_{\varsigma=s_{0}-3}^{s_{0}} \widehat{J}_{\varsigma}^{+}\left(\tau, \lambda\left(s^{\mathbb{C}}\right)\right)-\left(\left|J_{s_{0}-1}\right|-s_{0}^{2}\right) \widehat{J}_{s_{0}}^{+}\left(\tau, \lambda\left(s^{\mathbb{C}}\right)\right)\right] \\
& \leq(n+4) \Upsilon_{2}^{t} 2^{-q s_{0}^{2}} \sum_{\varsigma=s_{0}-3}^{s_{0}} \widehat{J}_{\varsigma}^{-}\left(\tau, \lambda\left(s^{\mathbb{C}}\right)\right) .
\end{aligned}
$$

Together with (4.17) we get

$$
\left|\widehat{\rho}-\widehat{\rho}^{\prime}\right| \leq 3(n+4)\left(\Upsilon_{1} \Upsilon_{2}\right)^{t} 2^{-q s_{0}^{2}}\left|\widehat{\rho}^{\prime}\right| .
$$

Putting $C=3(n+4)\left(\Upsilon_{1} \Upsilon_{2}\right)^{t_{*, \text { sup }}}$ we have

$$
\frac{\left|\widehat{\rho}-\widehat{\rho}^{\prime}\right|}{|\widehat{\rho}|} \leq \frac{\left|\widehat{\rho}-\widehat{\rho}^{\prime}\right|}{\left|\widehat{\rho}^{\prime}\right|} \leq C 2^{-q s_{0}^{2}} \leq C \exp \left(-q s_{0}^{2} \log 2\right) .
$$

3. Using the inequalities $s_{0} \geq s^{\mathbb{C}} \geq s^{+}(\tau)-2, s^{+}\left(\tau_{0}\right) \geq 20, \Xi-2 \xi \geq 1$, and the

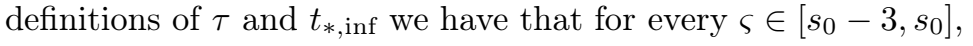

$$
\varsigma^{2} \geq \frac{s_{0}^{2}}{2} \geq \frac{\left(s^{+}(\tau)-2\right)^{2}}{2} \geq \frac{\left(s^{+}(\tau)\right)^{2}}{4}=\frac{\Xi-2 \xi}{4 q(1-\tau)} \geq \frac{t_{*, \mathrm{inf}}}{4 q\left(t_{*}-t\right)} .
$$

Using (4.6) and putting $v_{!}:=v_{0} \frac{t_{*, \text { inf }}}{8 q}$, we get

$$
C_{0} \exp \left(\frac{-v_{0} \cdot t_{*, \text { inf }}}{4 q\left(t_{*}-t\right)}\right) \leq \exp \left(\frac{-v_{!}}{t_{*}-t}\right) .
$$


For $\varsigma \in\{+,-\}$, denote by $\mathcal{O}^{\varsigma}$ the forward orbit of $p^{\varsigma}$ under $f$. Recall that $\tau_{m_{0}-1} \leq \tau \leq \tau_{m_{0}}$ and $s^{\mathbb{C}}$ in $\left[s^{+}(\tau)-2, s^{-}(\tau)+\Omega\right]$. Using that $s^{+}$and $s^{-}$are increasing functions we have

$$
s^{+}\left(\tau_{m_{0}-1}\right)-2<s^{+}(\tau)-2 \leq s^{\mathbb{C}} \leq s^{-}(\tau)+\Omega \leq s^{-}\left(\tau_{m_{0}}\right)+\Omega .
$$

Hence,

and by 4.7 we

$$
s^{+}\left(\tau_{m_{0}-1}\right)-2<s_{0} \leq s^{-}\left(\tau_{m_{0}}\right)+\Omega+1 .
$$

$$
s^{+}\left(\tau_{m_{0}-1}\right)-2<s_{0}<s^{+}\left(\tau_{m_{0}+1}\right)-5 .
$$

This implies that

$$
\left[s_{0}-3, s_{0}\right] \subseteq\left[\ell\left(m_{0}-1\right), \ell\left(m_{0}+1\right)\right) .
$$

A similar argument shows that if $\tau=\tau_{m_{0}-1}$ then

$$
\left[s_{0}-3, s_{0}\right] \subseteq\left[\ell\left(m_{0}-1\right), \ell\left(m_{0}\right)\right) .
$$

Fix an integer $\varsigma$ in $\left[s_{0}-3, s_{0}\right]$ and let $m(\varsigma)$ in $\mathbb{N}_{0}$ be so that $\ell(m(\varsigma)) \leq \varsigma<\ell(m(\varsigma)+1)$. For every integer $j$ such that $j+1$ is in $\bigcup_{k \in[\ell(m(\varsigma)), \ell(m(\varsigma)+1))} J_{k}$ we have $\underline{\widehat{x}}(\underline{\varsigma})_{j}=$ $1^{\varsigma(m(\varsigma))}$, so

$$
\iota(\underline{\varsigma})_{j}= \begin{cases}1 & \text { if } \varsigma(m(\varsigma))=+; \\ 0 & \text { if } \varsigma(m(\varsigma))=- \text { and } j \text { is even; } \\ 1 & \text { if } \varsigma(m(\varsigma))=- \text { and } j \text { is odd. }\end{cases}
$$

Since $b_{\varsigma}$ is even, for every $\ell$ in $\left[0, a_{\varsigma+1}-1-b_{\varsigma}\right]$ the points $f^{n+1+3\left(b_{\varsigma}+\ell-1\right)}(0)$ and $f^{3 \ell}\left(p^{\varsigma(m(\varsigma))}\right)$ are both in $Y_{f}$ or both in $\widetilde{Y}_{f}$. Since $Y_{f}$ and $\tilde{Y}_{f}$ are puzzle pieces of depth 4 , it follows that

$$
P_{f, 3\left(a_{\varsigma+1}-1-b_{\varsigma}\right)+4}\left(f^{n+1+3\left(b_{\varsigma}-1\right)}(0)\right)=P_{f, 3\left(a_{\varsigma+1}-1-b_{\varsigma}\right)+4}\left(p^{\varsigma(m(\varsigma))}\right) .
$$

Then, for each integer $j$ in $\left[b_{\varsigma}-1, a_{\varsigma+1}-2\right]$ we have

$$
P_{f, 3\left(a_{\varsigma}+1-2-j\right)+4}\left(f^{n+1+3 j}(0)\right)=P_{f, 3\left(a_{\varsigma}+1-2-j\right)+4}\left(f^{3 j}\left(p^{\varsigma(m(\varsigma))}\right)\right),
$$

which implies that for each integer $k$ in $J_{\varsigma}$ and for each integer $j$ in $\left[b_{\varsigma}-1, k-1\right]$,

$$
P_{f, 3(k-j)+1}\left(f^{n+1+3 j}(0)\right)=P_{f, 3(k-j)+1}\left(f^{3 j}\left(p^{\varsigma(m(\varsigma))}\right)\right) .
$$

Note that by definition of $\mathfrak{D}_{k}$, every element $W$ of $\mathfrak{D}_{k}$ is contained in $P_{f, n+3 k+2}(0)$, so, if in addition we have $k \geq b_{\varsigma}+\varsigma^{2}$ and $j \leq k-\varsigma^{2}$, then by (4.20) and Lemma.2 we obtain

$f^{n+1+3 j}(W) \cup f^{(n+1+3 j)+1}(W) \cup f^{(n+1+3 j)+2}(W) \subset B\left(\mathcal{O}^{\varsigma(m(\varsigma))}, \exp \left(-v_{!} /\left(t_{*}-t\right)\right)\right)$, which proves that $\widehat{\rho}_{\varsigma}^{\prime}$ is supported on $B\left(\mathcal{O}^{\varsigma}(m(\varsigma)), \exp \left(-v_{!} /\left(t_{*}-t\right)\right)\right)$.

On the other hand, for each integer $k$ in $J_{s_{0}}$, every element $W$ of $\mathfrak{D}_{k}$ is contained in $P_{f, n+3 k+2}(0)$, and hence in $P_{f, n+3\left(a_{s_{0}}-1\right)+2}(0)$. Thus, by (4.23) with $\varsigma=s_{0}-1$ we have, by (4.20) and Lemma A.2 that for every integer $j$ in $\left[b_{s_{0}-1}-1, a_{s_{0}}-s_{0}^{2}\right]$, $f^{n+1+3 j}(W) \cup f^{(n+1+3 j)+1}(W) \cup f^{(n+1+3 j)+2}(W) \subset B\left(\mathcal{O}^{\varsigma\left(m\left(s_{0}-1\right)\right)}, \exp \left(-v_{!} /\left(t_{*}-t\right)\right)\right)$, which proves that $\widehat{\rho}^{\prime \prime}$ is supported on $B\left(\mathcal{O}^{\varsigma}\left(m\left(s_{0}-1\right)\right), \exp \left(-v_{!} /\left(t_{*}-t\right)\right)\right)$.

Let $m$ and $\widehat{m}$ be integers as in the statement of Theorem $\mathrm{B}$, that is,

$$
\widehat{m} \geq m \geq 1, \varsigma(m)=\cdots=\varsigma(\widehat{m}) \text {, and fix } t \text { in }\left[t_{*} \tau_{m}, t_{*} \tau_{\widehat{m}}\right] .
$$

Then, $\tau_{m} \leq \tau \leq \tau_{\widehat{m}}$ so for every $\varsigma$ in $\left[s_{0}-3, s_{0}\right]$, by (4.21) if $\widehat{m}>m$ and by (4.22) if $\widehat{m}=m$, we have $\varsigma(m(\varsigma))=\varsigma(m)$. It follows from the considerations above that 
the measure $\widehat{\rho}^{\prime}$ is supported on $B\left(\mathcal{O}^{\varsigma(m)}, \exp \left(-v_{!} /\left(t_{*}-t\right)\right)\right)$. To conclude the proof, recall that the equilibrium state $\rho_{t}$ of $\left.f\right|_{J(f)}$ for the potential $-t \log |D f|$ is the probability measure proportional to $\hat{\rho}$. By (4.18) together with (4.19) and (4.20), we conclude that

$$
\rho_{t}\left(\mathbb{C} \backslash B\left(\mathcal{O}^{\varsigma(m)}, \exp \left(-\frac{v_{!}}{t_{*}-t}\right)\right)\right) \leq \frac{\left|\widehat{\rho}-\widehat{\rho}^{\prime}\right|}{|\widehat{\rho}|} \leq C \exp \left(-\frac{t_{*, \inf } \cdot \log 2}{2\left(t_{*}-t\right)}\right) .
$$

Recalling that $v_{!}=v_{0} \cdot t_{* \text {,inf }} / 8 q$, this completes the proof of Theorem $\mathrm{B}$ with

$$
\varkappa=\min \left\{\frac{v_{0}}{8 q}, \frac{\log 2}{2}\right\} t_{*, \text { inf }} .
$$

Remark 4.1. Without assuming the existence of $\widehat{m}$ and $m$ satisfying (4.24) the measure $\widehat{\rho}^{\prime}$ is supported on $B\left(\mathcal{O}^{+} \cup \mathcal{O}^{-}, \exp \left(-v_{!} /\left(t_{*}-t\right)\right)\right)$, and the estimate above gives that for every $t \in\left(t_{*} \tau_{1}, t_{*}\right)$ we have

$$
\rho_{t}\left(\mathbb{C} \backslash B\left(\mathcal{O}^{+} \cup \mathcal{O}^{-}, \exp \left(-\frac{\varkappa}{t_{*}-t}\right)\right)\right) \leq C \exp \left(-\frac{\varkappa}{t_{*}-t}\right) .
$$

\section{Appendix A. Estimating the GeOmetric Pressure FunCtion}

In this appendix we collect several results from [CR17] that we use in the proofs Theorems $\mathrm{A}$ and $\mathrm{B}$ in $\$ 4$

Throughout this section we fix a uniform family of quadratic-like maps $\mathscr{F}$, with constants $R$ and $K$.

A.1. Some uniform bounds. For a parameter $c$ in $\mathcal{P}_{2}(-2)$ the external rays $R_{c}(7 / 24)$ and $R_{c}(17 / 24)$ land at the point $\gamma(c)$ in $P_{c, 1}(0)$, see CRL13, §3.3]. Let $\widehat{U}_{c}$ be the open disk containing $-\beta(c)$ that is bounded by the equipotential 2 and by

$$
R_{c}(7 / 24) \cup\{\gamma(c)\} \cup R_{c}(17 / 24) .
$$

Put $\widehat{W}_{c}:=f_{c}^{-1}\left(\widehat{U}_{c}\right)$, and for every $n \geq 3$ and every $f$ in $\mathcal{K}_{n}(\mathscr{F})$ put $\widehat{W}_{f}:=$ $h_{f}\left(\widehat{W}_{c(f)}\right)$.

Lemma A.1 (Uniform distortion bound, CR17], Lemma 4.9). There is $\Xi_{2}>1$ such that for each integer $n \geq 4$ and each $f$ in $\mathcal{K}_{n}(\mathscr{F})$ the following properties hold: For each integer $m \geq 1$ and each connected component $W$ of $f^{-m}\left(P_{f, 1}(0)\right)$ on which $f^{m}$ is univalent, $f^{m}$ maps a neighborhood of $W$ biholomorphically to $\widehat{W}_{f}$ and the distortion of this map on $W$ is bounded by $\Xi_{2}$.

Recall that $g_{f}: h_{f}\left(Y_{c(f)} \cup \tilde{Y}_{c(f)}\right) \rightarrow P_{f, 1}(0)$ is defined by $g_{f}=h_{f} \circ g_{c(f)} \circ h_{f}^{-1}$, see 3.1

Lemma A.2 ([CR17], Lemma 4.11). Let $\mathscr{F}$ be a uniform family of quadratic-like maps. Then, there are constants $C_{0}>0$ and $v_{0}>0$ such that for every $f$ in $\mathcal{P}_{5}(\mathscr{F})$, every $\ell$ in $\mathbb{N}$, and every connected component $W$ of $g_{f}^{-\ell}\left(P_{f, 1}(0)\right)$, we have

$$
\max \left\{\operatorname{diam}(W), \operatorname{diam}(f(W)), \operatorname{diam}\left(f^{2}(W)\right)\right\} \leq C_{0} \exp \left(-v_{0} \ell\right) .
$$

A.2. Geometric Peierls condition. Let $n \geq 5$ be an integer and $f$ in $\mathcal{K}_{n}(\mathscr{F})$. Put

$$
D_{f}^{\prime}:=\left\{w \in \mathbb{C} \backslash V_{f} \mid f^{m}(w) \in V_{f} \text { for some } m \in \mathbb{N}\right\},
$$

and for $w$ in $D_{f}^{\prime}$ denote by $m_{f}(w)$ the least integer $m$ such that $f^{m}(w) \in V_{f}$ and call it the first landing time of $w$ to $V_{f}$. The first landing map to $V_{f}$ is the $\operatorname{map} L_{f}: D_{f}^{\prime} \rightarrow V_{f}$ defined by $L_{f}(w):=f^{m_{f}(w)}(w)$. 
Definition A.3 (Geometric Peierls Condition). Given $\kappa>0$ and $v>0$, a quadraticlike map $f$ in $\mathscr{F}$ satisfies the Geometric Peierls Condition with constants $\kappa$ and $v$, if for every $z$ in $L_{f}^{-1}\left(V_{f}\right)$ we have

$$
\left|D L_{f}(z)\right| \geq \kappa \exp \left(\left(\chi_{\text {crit }}(f) / 2+v\right) m_{f}(z)\right) .
$$

Proposition A.4 ([CR17, Proposition 4.3). For every $v>0$ satisfying $v<\frac{1}{2} \log 2$ and every $R^{\prime}>0$, there are constants $K_{1}>1, n_{1} \geq 6$, and $\kappa_{1}>0$, such that the following property holds. If $\mathscr{F}_{1}$ admits $K_{1}$ and $R^{\prime}$ as uniformity constants, then for every integer $n \geq n_{1}$, every element $f$ of $\mathcal{K}_{n}\left(\mathscr{F}_{1}\right)$ satisfies the Geometric Peierls Condition with constants $\kappa_{1}$ and $v$, and we have

$$
\chi_{f}(\beta(f))>\chi_{\text {crit }}(f)+2 v,
$$

$$
\chi_{\text {crit }}(f)>2 v \text {, and } \chi_{f}(p(f))<\chi_{f}\left(p^{+}(f)\right)+v / 4 \text {. }
$$

\section{A.3. Controlling the geometric pressure function via the postcritical se-} ries.

Proposition A.5 ([CR17], Proposition I). Let $\mathscr{F}$ be a uniform family of normalized quadratic-like maps. For every $\kappa>0$ and every $v>0$ there are $n_{2} \geq 5$ and $C_{1}>1$ such that for every integer $n \geq n_{2}$ and every $f$ in $\mathcal{K}_{n}(\mathscr{F})$ satisfying the Geometric Peierls Condition with constants $\kappa$ and $v$, the following properties hold for each $t \geq 2 \log 2 / v$.

1. For $p$ in $\left[-t \chi_{\text {crit }}(f) / 2,0\right)$ satisfying

$$
\sum_{k=0}^{+\infty} \exp (-(n+3 k) p)\left|D f^{n+3 k}(f(0))\right|^{-t / 2} \geq C_{1}^{t},
$$

we have $\mathscr{P}_{f}^{\mathbb{R}}(t, p)>0$ and $P_{f}^{\mathbb{R}}(t) \geq p$. If in addition the sum above is finite, then $\mathscr{P}_{f}(t, p)$ is finite and $P_{f}^{\mathbb{R}}(t)>p$.

2. For $p \geq-t \chi_{\text {crit }}(f) / 2$ satisfying

$$
\sum_{k=0}^{+\infty} \exp (-(n+3 k) p)\left|D f^{n+3 k}(f(0))\right|^{-t / 2} \leq C_{1}^{-t}
$$

we have $\mathscr{P}_{f}(t, p)<0$ and $P_{f}(t) \leq p$.

3. For $p \geq-t \chi_{\text {crit }}(f) / 2$ satisfying

$$
\sum_{k=0}^{+\infty} k \cdot \exp (-(n+3 k) p)\left|D f^{n+3 k}(f(0))\right|^{-t / 2}<+\infty
$$

we have

$$
\sum_{W \in \mathfrak{D}_{f}} m_{f}(W) \cdot \exp \left(-m_{f}(W) p\right) \sup _{z \in W}\left|D F_{f}(z)\right|^{-t}<+\infty .
$$

Let $n \geq 4$ be an integer and $f$ in $\mathcal{K}_{n}(\mathscr{F})$. Since the critical point $z=0$ does not belong to $D_{f}$ (cf., Lemma 4.2 in [CRL13]), for each integer $\ell \geq 1$, each connected component of $D_{f}$ intersecting $P_{f, \ell}(0)$ is contained in $P_{f, \ell}(0)$. We define the level of a connected component $W$ of $D_{f}$ as the largest integer $k \geq 0$ such that $W$ is contained in $P_{f, n+3 k+2}(0)$. Given an integer $k \geq 0$ denote by $\mathfrak{D}_{f, k}$ the collection of all connected components of $D_{f}$ of level $k$; we have $\mathfrak{D}_{f}=\bigcup_{k=0}^{+\infty} \mathfrak{D}_{f, k}$. 
Proposition A.6 ([CR17], Proposition 5.6). Let $\mathscr{F}$ be a uniform family of normalized quadratic-like maps. For every $\kappa>0$ and every $v>0$ there are $n_{3} \geq 5$ and $C_{2}>1$ such that for every integer $n \geq n_{3}$ and every $f$ in $\mathcal{K}_{n}(\mathscr{F})$ satisfying the Geometric Peierls Condition with constants $\kappa$ and $v$, the following properties hold for each $t \geq 2 \log 2 / v$ and each integer $k \geq 0$ :

1. For each $p<0$, we have

$$
\begin{aligned}
\sum_{W \in \mathfrak{D}_{f, k} \cap \mathfrak{D}_{f}^{\mathbb{R}}} \exp \left(-m_{f}(W) p\right) \inf _{z \in W}\left|D F_{f}(z)\right|^{-t}> & \\
& C_{2}^{-t} \exp (-(n+3 k) p)\left|D f^{n+3 k}(f(0))\right|^{-t / 2} .
\end{aligned}
$$

2. For each $p \geq-t \chi_{\mathrm{crit}}(f) / 2-t v / 3$, we have

$$
\begin{aligned}
\sum_{W \in \mathfrak{D}_{f, k}} \exp \left(-m_{f}(W) p\right) \sup _{z \in W}\left|D F_{f}(z)\right|^{-t}< & \\
& C_{2}^{t} \exp (-(n+3 k) p)\left|D f^{n+3 k}(f(0))\right|^{-t / 2} .
\end{aligned}
$$

Moreover,

$$
\begin{aligned}
\sum_{W \in \mathfrak{D}_{f, k}}\left(m_{f}(W)-(n+3 k)\right) \exp \left(-m_{f}(W) p\right) \sup _{z \in W}\left|D F_{f}(z)\right|^{-t}< \\
C_{2}^{t} \exp (-(n+3 k) p)\left|D f^{n+3 k}(f(0))\right|^{-t / 2} .
\end{aligned}
$$

A.4. Estimating the postcritical series. Denote by $\widehat{\Sigma}$ the set of all those sequences $\left(\widehat{x}_{j}\right)_{j \in \mathbb{N}_{0}}$ in $\left\{0,1^{+}, 1^{-}\right\}^{\mathbb{N}_{0}}$ such that for each $j$ in $\mathbb{N}_{0}$ satisfying $\widehat{x}_{j}=1^{+}$ (resp. $\left.\widehat{x}_{j}=1^{-}\right)$, we have $\widehat{x}_{j+1} \neq 1^{-}\left(\right.$resp. $\left.\widehat{x}_{j+1} \neq 1^{+}\right)$. A sequence $\left(x_{j}\right)_{j \in \mathbb{N}_{0}}$ in $\{0,1\}^{\mathbb{N}_{0}}$ is compatible with a sequence $\left(\widehat{x}_{j}\right)_{j \in \mathbb{N}_{0}}$ in $\widehat{\Sigma}$ if for every $j$ in $\mathbb{N}_{0}$ such that $\widehat{x}_{j}=0$ (resp. $\left.\widehat{x}_{j}=1^{+}, \widehat{x}_{j}=\widehat{x}_{j+1}=1^{-}\right)$, we have $x_{j}=0\left(\right.$ resp. $x_{j}=1$, $\left.x_{j} \neq x_{j+1}\right)$.

Fix a sequence $\left(\widehat{x}_{j}\right)_{j \in \mathbb{N}_{0}}$ in $\widehat{\Sigma}$. Define $N: \mathbb{N}_{0} \rightarrow \mathbb{N}_{0}$ by $N(0):=0$, and for $k$ in $\mathbb{N}$ by

$$
N(k):=\sharp\left\{j \in\{0, \ldots, k-1\} \mid \widehat{x}_{j}=0\right\} .
$$

Moreover, define $B: \mathbb{N}_{0} \rightarrow \mathbb{N}_{0}$ by $B(0):=0, B(1):=1$, and for $k \geq 2$ by

$$
B(k):=1+\sharp\left\{j \in\{0, \ldots, k-2\} \mid \widehat{x}_{j} \neq \widehat{x}_{j+1}\right\} .
$$

Note that for $k$ in $\mathbb{N}$ the function $B(k)$ is equal to the number of blocks of 0 's, $1^{+}$'s, and $1^{-}$'s in the sequence $\left(\widehat{x}_{j}\right)_{j=0}^{k-1}$.

Throughout the rest of this subsection, fix a uniform family of quadratic-like maps $\mathscr{F}$, and let $\Xi_{2}>1$ be the constant given by Lemma A.1. Note that for $n \geq 5$ and $f$ in $\mathcal{K}_{n}(\mathscr{F})$, the condition $\theta(f)>1$ is equivalent to $\chi_{f}(p(f))>\chi_{f}\left(p^{+}(f)\right)$, see (3.7) for the definition of $\theta(f)$. When this holds, define

$$
\xi(f):=\frac{\log \Xi_{2}}{2 \log \theta(f)} .
$$

If in addition the itinerary $\iota(f)$ is compatible with $\left(\widehat{x}_{j}\right)_{j \in \mathbb{N}_{0}}$, let $\xi \geq \xi(f)$ be given and define for each integer $k \geq 0$ and each $(\tau, \lambda)$ in $[0,+\infty) \times[0,+\infty)$,

$$
\pi_{f, k}^{ \pm}(\tau, \lambda):=2^{-\lambda k-\tau N(k) \pm \xi \tau B(k)} .
$$


Lemma A.7 ([CR17, Lemma 6.1). There is $\Xi_{3}>1$ such that the following property holds. Let $\underline{\widehat{x}}$ be a sequence in $\widehat{\Sigma}$ such that $N(k) / k \rightarrow 0$ as $k \rightarrow+\infty$, and such that the length of every maximal block containing only $1^{-}$'s is even. Then, for every integer $n \geq 6$ and every $f$ in $\mathcal{K}_{n}(\mathscr{F})$ such that $\chi\left(p^{-}(f)\right)=\chi\left(p^{+}(f)\right)$, and such that the itinerary $\iota(f)$ is compatible with $\underline{\hat{x}}$, we have

$$
\chi_{\text {crit }}(f)=\frac{1}{3} \log \left|D g_{f}\left(p^{+}(f)\right)\right| .
$$

If moreover

$$
\chi_{f}(p(f))>\chi_{f}\left(p^{+}(f)\right)
$$

the following property holds for every choice of $\xi \geq \xi(f)$. For every integer $k \geq 0$, and all $t>0$ and $\delta \geq 0$, we have

$$
\begin{aligned}
& \Xi_{3}^{-\frac{t}{2}} \exp (-n \delta)\left(\frac{\exp \left(\chi_{\text {crit }}(f)\right)}{|D f(\beta(f))|}\right)^{\frac{t}{2} n} \pi_{f, k}^{-}\left(\frac{\log \theta(f)}{\log 2} t, \frac{3 \delta}{\log 2}\right) \\
& \quad \leq \exp \left(-(n+3 k)\left(-t \frac{\chi_{\text {crit }}(f)}{2}+\delta\right)\right)\left|D f^{n+3 k}(f(0))\right|^{-\frac{t}{2}} \\
& \leq \Xi_{3}^{\frac{t}{2}} \exp (-n \delta)\left(\frac{\exp \left(\chi_{\text {crit }}(f)\right)}{|D f(\beta(f))|}\right)^{\frac{t}{2} n} \pi_{f, k}^{+}\left(\frac{\log \theta(f)}{\log 2} t, \frac{3 \delta}{\log 2}\right) .
\end{aligned}
$$

Appendix B. Estimating the positive temperature 2 VARIAbles SERIES

B.1. Preliminary estimates. Given an integer $\Xi \geq 0$, let $q \geq 100(\Xi+1)$ be a sufficiently large integer such that $2^{q-3} \geq q+1+\Xi$. Define the cubic function

$$
\begin{aligned}
Q: \mathbb{R} & \rightarrow \mathbb{R} \\
s & \mapsto Q(s):=q s^{3} .
\end{aligned}
$$

For each real number $s$ in $[0,+\infty)$ define

$$
a_{s}:=2^{Q(s)} \text { and } b_{s}:=2^{Q(s)}+Q(s+1)-Q(s)+\Xi,
$$

and the following intervals of $\mathbb{R}$ :

$$
I_{s}:=\left[a_{s}, b_{s}\right) \text { and } J_{s}:=\left[b_{s}, a_{s+1}\right) .
$$

Note that $\left|I_{0}\right|=q+\Xi$, and that for integer values of $s$, the intervals $I_{s}$ and $J_{s}$ form a partition of $[1,+\infty)$ that we use in $\$ 4.1$ to define a certain family of itineraries. For $s$ in $[0,+\infty)$ that is not necessarily an integer, the interval $J_{s}$ is used in the proof of Lemmas B.2 and B.4 in $\$ \mathrm{~B} .2$ and $\$ \mathrm{~B} .3$ respectively.

Define the function $N: \mathbb{N}_{0} \rightarrow \mathbb{N}_{0}$, by $N(0):=0$, and for $k$ in $\mathbb{N}$ by

$$
N(k):=\sharp\left\{j \in\{0, \ldots, k-1\} \mid j+1 \in \bigcup_{s=0}^{+\infty} I_{s}\right\},
$$

and the function $B: \mathbb{N}_{0} \rightarrow \mathbb{N}_{0}$ by $B(0):=0$, and for $s$ in $\mathbb{N}_{0}$ by

$$
B^{-1}(2 s+1)=I_{s} \quad \text { and } \quad B^{-1}(2(s+1))=J_{s} .
$$

Observe that for every $s$ in $\mathbb{N}_{0}$, we have for every $k$ in $J_{s}$

$$
N(k)=\sum_{j=0}^{s}\left|I_{j}\right|=\sum_{j=0}^{s}(Q(j+1)-Q(j)+\Xi)=Q(s+1)+\Xi \cdot(s+1)
$$


and for every $k$ in $I_{s}$

$$
N(k)=k+1-a_{s}+Q(s)+\Xi s .
$$

Let $\xi>0$ be given, put $\Xi:=\lceil 2 \xi\rceil+1$, and let $q$ be as before. For $s$ in $\mathbb{N}_{0}$ define the following 2 variables series on $[0,+\infty) \times[0,+\infty)$,

$$
I_{s}^{ \pm}(\tau, \lambda):=\sum_{k \in I_{s}} 2^{-\lambda k-\tau N(k) \pm \tau \xi B(k)} \quad \text { and } \quad J_{s}^{ \pm}(\tau, \lambda):=\sum_{k \in J_{s}} 2^{-\lambda k-\tau N(k) \pm \tau \xi B(k)},
$$

and put

$$
\Pi^{ \pm}(\tau, \lambda):=1+\sum_{s=0}^{+\infty} I_{s}^{ \pm}(\tau, \lambda)+\sum_{s=0}^{+\infty} J_{s}^{ \pm}(\tau, \lambda) .
$$

Note that by (B.1) and (B.2), for all $j$ in $\mathbb{N}_{0}$ and all $\tau>0$ we have

$$
J_{j}^{ \pm}(\tau, \lambda)=2^{-\tau Q(j+1)-(\Xi \mp 2 \xi) \tau \cdot(j+1)} \sum_{k \in J_{j}} 2^{-\lambda k} .
$$

For every real number $s$ in $[0,+\infty)$ define

$$
\lambda(s):=\frac{1}{\left|J_{s}\right|} .
$$

By part 2 of Lemma B.1 (below) and the hypothesis $q \geq 100(\Xi+1)$, we have

$$
0<\lambda(s) \leq 1 / 4 \text {. }
$$

In $₫ \mathrm{~B} .2$ we estimate $\Pi^{+}$from above and $\Pi^{-}$from below for some particular values of the variable $\lambda$ depending on the variable $\tau$. In $\$ \mathrm{~B} .3$ we estimate a weighted version of the series $\Pi^{+}$from above.

We finish this subsection with a lemma collecting some simple estimates that are extensively used in what follows.

Lemma B.1. The following properties hold:

1. For each real number $s \geq 0$, we have $b_{s} \leq a_{s+1} / 2$ and the function $\lambda(s)$ is strictly decreasing;

2. For each real number $s \geq 0$, we have $a_{s+1} / 2 \leq\left|J_{s}\right|$;

3. For every $s \geq 1$, we have $b_{s}+2(s+1)^{2} \leq \frac{5}{4} a_{s}$;

4. Given $s \geq 1$, put $s_{0}:=\lceil s\rceil$. Then, we have $b_{s_{0}}+2 s_{0}^{2} \leq 3\left|J_{s}\right|$.

Proof. Part 1 , case $s=0$ is given by our hypothesis $2^{q-3} \geq q+1+\Xi$. For $s>0$, it follows from this and from the fact that, by the hypothesis $q \geq 100(\Xi+1)$, the derivative of the function

$$
s \mapsto 2^{q(s+1)^{3}-1}-\left(2^{q s^{3}}+q\left(3 s^{2}+3 s+1\right)+\Xi\right)
$$

is strictly positive on $[0,+\infty)$. This also implies that $\lambda(s)$ is strictly decreasing.

Part 2 follows from part 1.

Part 3, case $s=1$ is given by our hypotheses $q \geq 100(\Xi+1)$ and $2^{q-3} \geq q+1+\Xi$. For $s>1$, it follows from this and from the fact that the derivative of the function

$$
s \mapsto \frac{5}{4} 2^{q s^{3}}-\left(2^{q s^{3}}+q\left(3 s^{2}+3 s+1\right)+\Xi+2(s+1)^{2}\right)
$$

is strictly positive on $[1,+\infty)$.

Part 4 follows from parts 2 and 3. 
B.2. Estimating the 2 variables series. Define the functions $s^{+}, s^{-}:(-\infty, 1) \rightarrow$ $\mathbb{R}$ by

$$
s^{ \pm}(\tau):=\left(\frac{\Xi \mp 2 \xi}{q(1-\tau)}\right)^{\frac{1}{2}} .
$$

Lemma B.2. For every $\tau$ in $\left(\frac{q-1}{q}, 1\right)$, we have

$$
\Pi^{+}\left(\tau, \lambda\left(s^{+}(\tau)-2\right)\right) \leq 25+5 \cdot 2^{\tau \xi}
$$

and for every $\Omega \geq 0$, we have

$$
2^{2 \Omega-3} \leq \Pi^{-}\left(\tau, \lambda\left(s^{-}(\tau)+\Omega\right)\right) .
$$

Sublemma B.3. The following properties hold:

1. For $\tau \geq 1$ we have

$$
\Pi^{+}(\tau, 0) \leq 2\left(2^{\tau \xi}+1\right) .
$$

2. For every real number $s$ in $[0,+\infty)$ and every $\tau$ in $(2 / 3,1)$ satisfying $\tau>$ $\frac{Q(s+1)-1}{Q(s+2)}$, we have

$$
\Pi^{+}(\tau, \lambda(s)) \leq 4+5 \cdot 2^{\tau \xi}+2 \sum_{j=0}^{\lfloor s\rfloor+1} 2^{(1-\tau) Q(j+1)-\tau(\Xi-2 \xi)(j+1)} .
$$

3. For every real number $s$ in $[0,+\infty)$ and every $\tau>0$, we have

$$
\frac{1}{8} 2^{(1-\tau) Q(\lfloor s\rfloor+1)-\tau(\Xi+2 \xi)(\lfloor s\rfloor+1)} \leq \Pi^{-}(\tau, \lambda(s)) .
$$

Proof. 1. By (B.1), (B.3), and the hypothesis $\Xi-2 \xi \geq 1$, for every $\tau>0$ and every $\lambda \geq 0$ we have

$$
\begin{aligned}
\sum_{s=0}^{+\infty} I_{s}^{+}(\tau, \lambda) & \leq \sum_{s=0}^{+\infty} \sum_{m=1}^{\left|I_{s}\right|} 2^{-\tau(Q(s)+\Xi s+m)+\tau \xi \cdot(2 s+1)} \\
& =2^{\tau \xi} \sum_{s=0}^{+\infty} 2^{-\tau(Q(s)+(\Xi-2 \xi) s)} \sum_{m=1}^{\left|I_{s}\right|} 2^{-\tau m} \\
& \leq 2^{\tau \xi} \frac{2^{-\tau}}{1-2^{-\tau}} \sum_{s=0}^{+\infty} 2^{-\tau(\Xi-2 \xi) s} \\
& \leq 2^{\tau \xi} \frac{2^{-\tau}}{\left(1-2^{-\tau}\right)^{2}} .
\end{aligned}
$$

By (B.1), (B.2), the hypothesis $\Xi-2 \xi \geq 1$, and that for every $s \geq 0$ we have $\left|J_{s}\right| \leq a_{s+1}$, we obtain for every $\tau \geq 1$ and with $\lambda=0$

$$
\begin{aligned}
\sum_{s=0}^{+\infty} J_{s}^{+}(\tau, 0) & =\sum_{s=0}^{+\infty}\left|J_{s}\right| 2^{-\tau(Q(s+1)+\Xi \cdot(s+1))+2 \tau \xi \cdot(s+1)} \\
& \leq \sum_{s=0}^{+\infty} 2^{-(\tau-1) Q(s+1)-\tau(\Xi-2 \xi)(s+1)} \\
& \leq \frac{2^{-\tau}}{1-2^{-\tau}}
\end{aligned}
$$


Combining inequalities (B.6) and (B.7), we get for every $\tau \geq 1$

$$
\Pi^{+}(\tau, 0) \leq 1+2^{\tau \xi} \frac{2^{-\tau}}{\left(1-2^{-\tau}\right)^{2}}+\frac{2^{-\tau}}{1-2^{-\tau}} \leq 2\left(2^{\tau \xi}+1\right) .
$$

This is part 1 of the sublemma.

2. Fix $s$ in $[0,+\infty)$ and set $s_{0}:=\lfloor s\rfloor$. We use (B.6) to estimate $\Pi^{+}(\tau, \lambda(s))$. To estimate $\sum_{j=0}^{+\infty} J_{j}^{+}(\tau, \lambda(s))$, note that by definition of $\lambda(s)$, for each integer $\ell$ satisfying $1 \leq \ell \leq\left|J_{s}\right|$ we have

$$
\frac{1}{2} \leq 2^{-\lambda(s) \ell} \leq 1
$$

On the other hand, the inequality $q \geq 100(\Xi+1)$ implies that the function $j \mapsto\left|J_{j}\right|$ is nondecreasing on $[0,+\infty)$. Therefore, for each $j$ in $\left\{0, \ldots, s_{0}\right\}$ we have $\left|J_{j}\right| \leq\left|J_{s}\right|$ and then

$$
\frac{1}{2}\left|J_{j}\right| \leq \sum_{m=1}^{\left|J_{j}\right|} 2^{-\lambda(s) m} \leq\left|J_{j}\right|
$$

On the other hand

$$
\sum_{m=1}^{+\infty} 2^{-\lambda(s) m}=\frac{1}{2^{\lambda(s)}-1} \leq \frac{1}{\lambda(s) \log 2} \leq 2\left|J_{s}\right| .
$$

Note also that for $j \in \mathbb{N}$, by (B.4), the inequalities $b_{j} \geq b_{0}=q+\Xi+1 \geq 1$, (B.9) and $\left|J_{s}\right| \leq 2^{Q(s+1)}$, we have

$$
\begin{aligned}
J_{j}^{+}(\tau, \lambda(s)) & =2^{-\tau(Q(j+1)+(\Xi-2 \xi)(j+1))} \sum_{k \in J_{j}} 2^{-\lambda(s) k} \\
& \leq 2\left|J_{s}\right| 2^{-\tau(Q(j+1)+(\Xi-2 \xi)(j+1))} \\
& \leq 2 \cdot 2^{Q(s+1)-\tau(Q(j+1)+(\Xi-2 \xi)(j+1))} .
\end{aligned}
$$

Taking $j=s_{0}+1$ and using the inequality $Q(s+1) \leq Q\left(s_{0}+2\right)$, we obtain,

$$
J_{s_{0}+1}^{+}(\tau, \lambda(s)) \leq 2 \cdot 2^{(1-\tau) Q\left(s_{0}+2\right)-\tau(\Xi-2 \xi)\left(s_{0}+2\right)} .
$$

On the other hand, our hypothesis $\tau>\frac{Q(s+1)-1}{Q(s+2)}$ implies that for $j \geq s_{0}+2$ we have

$$
Q(s+1)-\tau Q(j+1) \leq Q(s+1)-\tau Q(s+2)<1 .
$$

So, using the inequalities $\Xi-2 \xi \geq 1$ and $\tau>2 / 3$, and summing (B.10) over $j$ satisfying $j \geq s_{0}+2$, we obtain

$$
\sum_{j=s_{0}+2}^{+\infty} J_{j}^{+}(\tau, \lambda(s)) \leq \sum_{j=s_{0}+2}^{+\infty} 2^{2-\tau(\Xi-2 \xi)(j+1)} \leq \frac{2^{2-3 \tau}}{1-2^{-\tau}}<\frac{1}{1-2^{-\tau}} .
$$

Now we complete the estimate of $\sum_{j=0}^{+\infty} J_{j}^{+}(\tau, \lambda(s))$, by estimating the terms for which $j$ is in $\left\{0, \ldots, s_{0}\right\}$. From (B.8), (B.4), and $\left|J_{j}\right| \leq 2^{Q(j+1)}$, we deduce that for every integer $j$ in $\left\{0, \ldots, s_{0}\right\}$ we have

$$
J_{j}^{+}(\tau, \lambda(s)) \leq\left|J_{j}\right| \cdot 2^{-\tau(Q(j+1)+(\Xi-2 \xi)(j+1))} \leq 2^{(1-\tau) Q(j+1)-\tau(\Xi-2 \xi)(j+1)} .
$$


DANIEL CORONEL AND JUAN RIVERA-LETELIER

Summing over $j$ in $\left\{0, \ldots, s_{0}\right\}$ and using inequalities (B.11) and (B.12), we obtain

$$
\begin{aligned}
& \sum_{j=0}^{+\infty} J_{j}^{+}(\tau, \lambda(s)) \\
& \quad \leq \sum_{j=0}^{s_{0}} 2^{(1-\tau) Q(j+1)-\tau(\Xi-2 \xi)(j+1)}+2 \cdot 2^{(1-\tau) Q\left(s_{0}+2\right)-\tau(\Xi-2 \xi)\left(s_{0}+2\right)}+\frac{1}{1-2^{-\tau}} \\
& \quad \leq 2 \sum_{j=0}^{s_{0}+1} 2^{(1-\tau) Q(j+1)-\tau(\Xi-2 \xi)(j+1)}+\frac{1}{1-2^{-\tau}} .
\end{aligned}
$$

Together with (B.6) this implies

(B.13) $\Pi^{+}(\tau, \lambda(s))$

$$
\leq 1+\frac{2^{-\tau}}{\left(1-2^{-\tau}\right)^{2}} 2^{\tau \xi}+2 \sum_{j=0}^{s_{0}+1} 2^{(1-\tau) Q(j+1)-\tau(\Xi-2 \xi)(j+1)}+\frac{1}{1-2^{-\tau}} .
$$

Using that $\tau$ is in $(2 / 3,1)$, we have $\frac{1}{1-2^{-\tau}} \leq 3$ and $\frac{2^{-\tau}}{\left(1-2^{-\tau}\right)^{2}} \leq 5$. We obtain part 2 of the sublemma by combining these estimates with (B.13).

3. Fix $s$ in $[0,+\infty)$ and set $s_{0}:=\lfloor s\rfloor$. By parts 1 and 2 of Lemma B.1 we have

$$
\lambda(s)\left(b_{s_{0}}-1\right) \leq 2 \cdot \frac{b_{s_{0}}}{a_{s+1}} \leq 1 .
$$

By this inequality, part 2 of Lemma B.1, (B.4), the first inequality of (B.8), and the first equality in (B.10), we have

$$
\begin{aligned}
\frac{1}{2^{3}} 2^{(1-\tau) Q\left(s_{0}+1\right)-} & \tau(\Xi+2 \xi)\left(s_{0}+1\right) \\
& \leq \frac{1}{2^{2}}\left|J_{s_{0}}\right| 2^{-\tau\left(Q\left(s_{0}+1\right)+\Xi \cdot\left(s_{0}+1\right)\right)-2 \tau \xi \cdot\left(s_{0}+1\right)} \\
& \leq \frac{1}{2}\left|J_{s_{0}}\right| 2^{-\lambda(s)\left(b_{s_{0}}-1\right)-\tau\left(Q\left(s_{0}+1\right)+\Xi \cdot\left(s_{0}+1\right)\right)-2 \tau \xi \cdot\left(s_{0}+1\right)} \\
& \leq\left(\sum_{m=1}^{\left|J_{s_{0}}\right|} 2^{-\lambda(s) m}\right) 2^{-\lambda(s)\left(b_{s_{0}}-1\right)-\tau\left(Q\left(s_{0}+1\right)+\Xi \cdot\left(s_{0}+1\right)\right)-2 \tau \xi \cdot\left(s_{0}+1\right)} \\
& =J_{s_{0}}^{-}(\tau, \lambda(s)) .
\end{aligned}
$$

This proves part 3 of the sublemma and completes the proof.

Proof of Lemma B.2. Fix $\tau$ in $\left(\frac{q-1}{q}, 1\right)$ and $\Omega \geq 0$. Put $s(\tau)=\left(\frac{2}{q(1-\tau)}\right)^{\frac{1}{2}}$. Note that $\tau>\frac{q-1}{q} \Leftrightarrow 2<s(\tau)^{2}$. This last inequality and our hypothesis $q \geq 100(\Xi+1)$ imply that

$$
(3 q+2) s(\tau)<3 q s(\tau)^{2}+q+1 .
$$

Finally the last inequality is equivalent to

$$
\begin{aligned}
2 s(\tau) & <Q(s(\tau))-Q(s(\tau)-1)+1 \Leftrightarrow \\
(1-\tau) Q(s(\tau)) & <Q(s(\tau))-Q(s(\tau)-1)+1 \Leftrightarrow \\
\frac{Q(s(\tau)-1)-1}{Q(s(\tau))} & <\tau .
\end{aligned}
$$


On the other hand, the function $s \mapsto \frac{Q(s-1)-1}{Q(s)}$ is strictly increasing on $[0,+\infty)$. Using $1 \leq \Xi-2 \xi \leq 2$ we deduce

$$
\frac{Q\left(s^{+}(\tau)-1\right)-1}{Q\left(s^{+}(\tau)\right)} \leq \frac{Q(s(\tau)-1)-1}{Q(s(\tau))}<\tau .
$$

So the hypotheses of part 2 of Sublemma B.3 are satisfied with $s=s^{+}(\tau)-2$. Let $F: \mathbb{R} \rightarrow \mathbb{R}$ be the quadratic function defined by

$$
F(\ell):=(1-\tau) Q(\ell)-\tau(\Xi-2 \xi) \ell .
$$

Note that $F(0)=0$,

$$
F\left(\frac{s^{+}(\tau)}{2}\right)=\frac{(\Xi-2 \xi)^{\frac{3}{2}}}{8 q^{\frac{1}{2}}}(1-\tau)^{\frac{1}{2}}-\frac{3 \tau(\Xi-2 \xi)}{4}\left(\frac{s^{+}(\tau)}{2}\right)
$$

and

$$
F\left(s^{+}(\tau)\right)=\frac{(\Xi-2 \xi)^{\frac{3}{2}}}{q^{\frac{1}{2}}}(1-\tau)^{\frac{1}{2}} .
$$

Using that $F$ is convex on $[0,+\infty)$, we conclude that for each $\ell$ in $\left[0, s^{+}(\tau)\right]$ we have

$$
\begin{aligned}
F(\ell)=(1-\tau) Q(\ell)- & \tau(\Xi-2 \xi) \ell \\
\leq & \frac{(\Xi-2 \xi)^{\frac{3}{2}}}{q^{\frac{1}{2}}}(1-\tau)^{\frac{1}{2}}-\frac{3 \tau(\Xi-2 \xi)}{4} \min \left\{\ell, s^{+}(\tau)-\ell\right\} .
\end{aligned}
$$

Therefore, putting $s^{+}=s^{+}(\tau)-2$ and using $1 \leq \Xi-2 \xi \leq 2, q \geq 100(\Xi+1)$, and $\tau \geq \frac{q-1}{q}$, we have

$$
\begin{aligned}
\sum_{j=0}^{\left\lfloor s^{+}\right\rfloor+1} 2^{(1-\tau) Q(j+1)-\tau(\Xi-2 \xi)(j+1)} & \leq 2 \sum_{\ell=0}^{\left\lfloor s^{+}\right\rfloor+2} 2^{\frac{(\Xi-2 \xi)^{\frac{3}{2}}}{8 \sqrt{2}}(1-\tau)^{\frac{1}{2}}-\frac{3 \tau(\Xi-2 \xi)}{4} \ell} \\
& \leq 2 \cdot 2^{\frac{1}{40}} \frac{1}{1-2^{-\frac{3}{4} \cdot \frac{2}{3}}} \\
& <10 .
\end{aligned}
$$

The first inequality of the lemma is then obtained using part 2 of Sublemma B.3 with $s=s^{+}$.

To prove the second inequality, first we define $F^{-}: \mathbb{R} \rightarrow \mathbb{R}$ as the quadratic function given by

$$
F^{-}(\ell):=(1-\tau) Q(\ell)-\tau(\Xi+2 \xi) \ell .
$$

Notice from the definition of $\Xi=\lceil 2 \xi\rceil+1$ and the hypothesis that $\tau$ is in $\left(\frac{q-1}{q}, 1\right)$, we have

$$
\begin{aligned}
F^{-}\left(s^{-}(\tau)+\Omega\right)= & (1-\tau) \cdot(\Xi+2 \xi) s^{-}(\tau)+(3-\tau) \cdot(\Xi+2 \xi) \Omega \\
& +q(1-\tau) \Omega^{2}\left(3 s^{-}(\tau)+\Omega\right) \\
\geq & 2(\Xi+2 \xi) \Omega \\
\geq & 2 \Omega .
\end{aligned}
$$

Using that $F^{-}$is increasing on the interval $\left[\left(\frac{\tau}{3}\right)^{\frac{1}{2}} s^{-}(\tau),+\infty\right)$, that contains $s^{-}(\tau)$, and putting $s^{-}=s^{-}(\tau)+\Omega$, we get

$$
2 \Omega \leq F^{-}\left(s^{-}\right) \leq F^{-}\left(\left\lfloor s^{-}\right\rfloor+1\right)=(1-\tau) Q\left(\left\lfloor s^{-}\right\rfloor+1\right)-\tau(\Xi+2 \xi)\left(\left\lfloor s^{-}\right\rfloor+1\right) .
$$


Together with part 3 of Sublemma B.3 with $s=s^{-}$, we obtain

$$
2^{2 \Omega} \leq 2^{(1-\tau) Q\left(\left\lfloor s^{-}\right\rfloor+1\right)-\tau(\Xi+2 \xi)\left(\left\lfloor s^{-}\right\rfloor+1\right)} \leq 8 \Pi^{-}\left(\tau, \lambda\left(s^{-}\right)\right),
$$

proving the second inequality of the lemma.

B.3. Estimating the weighted 2 variables series. For each $s$ in $\mathbb{N}_{0}, \tau>0$, and $\lambda \geq 0$ put

$$
\begin{aligned}
& \widetilde{I}_{s}^{+}(\tau, \lambda):=\sum_{k \in I_{s}} k \cdot 2^{-\lambda k-\tau N(k)+\tau \xi B(k)}, \\
& \widetilde{J}_{s}^{+}(\tau, \lambda):=\sum_{k \in J_{s}} k \cdot 2^{-\lambda k-\tau N(k)+\tau \xi B(k)},
\end{aligned}
$$

and

$$
\widetilde{\Pi}^{+}(\tau, \lambda):=1+\sum_{s=0}^{+\infty} \widetilde{I}_{s}^{+}(\tau, \lambda)+\sum_{s=0}^{+\infty} \widetilde{J}_{s}^{+}(\tau, \lambda)
$$

Noting that by part 2 of Lemma B.1 we have $a_{s+1}-b_{s}=\left|J_{s}\right| \geq s^{2}+1$, define for each $\tau>0$ and $\lambda \geq 0$,

$$
\widehat{J}_{s}^{ \pm}(\tau, \lambda):=\sum_{k=b_{s}+s^{2}}^{a_{s+1}-1}\left(k+1-b_{s}-s^{2}\right) \cdot 2^{-\lambda k-\tau N(k) \pm \tau \xi B(k)} .
$$

Lemma B.4. For each $\tau$ in $[1 / 2,1]$, the following properties hold:

1. For each $s>0$ we have $\widetilde{\Pi}^{+}(\tau, \lambda(s))<+\infty$;

2. Given $s \geq 10$, put $s_{0}:=\lceil s\rceil$. Then

$$
\begin{array}{r}
\Pi^{+}(\tau, \lambda(s)) \leq \widetilde{\Pi}^{+}(\tau, \lambda(s))-\sum_{\varsigma=s_{0}-3}^{s_{0}} \widehat{J}_{\varsigma}^{+}(\tau, \lambda(s))-\left(\left|J_{s_{0}-1}\right|-s_{0}^{2}\right) J_{s_{0}}^{+}(\tau, \lambda(s)) \\
\leq 2^{-q s_{0}^{2}} \sum_{\varsigma=s_{0}-3}^{s_{0}} \widehat{J}_{\varsigma}^{-}(\tau, \lambda(s)) .
\end{array}
$$

The proof of this lemma is after the following lemma.

Sublemma B.5. Given $s \geq 10$, put $s_{0}:=\lceil s\rceil$. For every $\tau$ in $[1 / 2,1]$ the following properties hold:

1. $\widehat{J}_{s_{0}}^{-}(\tau, \lambda(s)) \geq \frac{1}{2^{9}} 2^{2 q(s+1)^{3}-q \tau \cdot\left(s_{0}+1\right)^{3}-(\Xi+2 \xi) \tau \cdot\left(s_{0}+1\right)}$;

2. For every integer $\varsigma$ in $\left[s_{0}-3, s_{0}-1\right]$ we have

$$
\left(b_{\varsigma}+\varsigma^{2}\right) J_{\varsigma}^{+}(\tau, \lambda(s)) \leq \frac{1}{20} 2^{-q s_{0}^{2}} \widehat{J}_{\varsigma}^{-}(\tau, \lambda(s)) ;
$$

3. $\left(b_{s_{0}}-\left|J_{s_{0}-1}\right|+2 s_{0}^{2}\right) J_{s_{0}}^{+}(\tau, \lambda(s)) \leq \frac{1}{4} 2^{-q s_{0}^{2}} \widehat{J}_{s_{0}}^{-}(\tau, \lambda(s))$. 
Proof. 1. By (B.1), (B.2), and part 4 of Lemma B.1 we have

$$
\begin{aligned}
\widehat{J}_{s_{0}}^{-}(\tau, \lambda(s)) & \\
& =2^{-q \tau \cdot\left(s_{0}+1\right)^{3}-(\Xi+2 \xi) \tau \cdot\left(s_{0}+1\right)} \sum_{k=b_{s_{0}}+s_{0}^{2}}^{a_{s_{0}+1}-1}\left(k+1-b_{s_{0}}-s_{0}^{2}\right) \cdot 2^{-\lambda(s) k} \\
& \geq \frac{1}{2^{3}} 2^{-q \tau \cdot\left(s_{0}+1\right)^{3}-(\Xi+2 \xi) \tau \cdot\left(s_{0}+1\right)} \sum_{m=1}^{\left|J_{s_{0}}\right|-s_{0}^{2}} m \cdot 2^{-\lambda(s) m} .
\end{aligned}
$$

Noticing that for every integer $N \geq 1$ we have

$$
\sum_{m=1}^{N} m \cdot 2^{-\lambda(s) m}=\frac{2^{\lambda(s)}}{\left(2^{\lambda(s)}-1\right)^{2}}\left(1-(N+1) 2^{-\lambda(s) N}+N 2^{-\lambda(s)(N+1)}\right),
$$

and that the function

$$
\eta \mapsto 1-(N+1) \eta^{N}+N \eta^{N+1}
$$

is decreasing on $[0,1]$, we have

$$
\begin{aligned}
& \sum_{m=1}^{\left|J_{s_{0}}\right|-s_{0}^{2}} m \cdot 2^{-\lambda(s) m} \\
= & \frac{2^{\lambda(s)}}{\left(2^{\lambda(s)}-1\right)^{2}} \cdot\left(1-\left(\left|J_{s_{0}}\right|-s_{0}^{2}+1\right) 2^{-\lambda\left(s_{0}\right)\left(\left|J_{s_{0}}\right|-s_{0}^{2}\right)}+\left(\left|J_{s_{0}}\right|-s_{0}^{2}\right) 2^{-\lambda\left(s_{0}\right)\left(\left|J_{s_{0}}\right|-s_{0}^{2}+1\right)}\right) \\
= & \frac{2^{\lambda(s)}}{\left(2^{\lambda(s)}-1\right)^{2}} \cdot\left(1-2^{\lambda\left(s_{0}\right) s_{0}^{2}-1}-\left(\left|J_{s_{0}}\right|-s_{0}^{2}\right) 2^{\lambda\left(s_{0}\right) s_{0}^{2}-1}\left(1-2^{-\lambda\left(s_{0}\right)}\right)\right) \\
= & \frac{2^{\lambda(s)}}{\left(2^{\lambda(s)}-1\right)^{2}} \cdot\left(1-2^{\lambda\left(s_{0}\right) s_{0}^{2}-1}\left(1+\left(\left|J_{s_{0}}\right|-s_{0}^{2}\right)\left(1-2^{-\lambda\left(s_{0}\right)}\right)\right)\right) .
\end{aligned}
$$

Observe that $1-2^{-\lambda\left(s_{0}\right)} \leq \log \left(2^{\lambda\left(s_{0}\right)}\right)$. Using this inequality we obtain

$$
\begin{aligned}
\sum_{m=1}^{\left|J_{s_{0}}\right|-s_{0}^{2}} m \cdot 2^{-\lambda(s) m} \geq \frac{2^{\lambda(s)}}{\left(2^{\lambda(s)}-1\right)^{2}}\left(1-2^{\lambda\left(s_{0}\right) s_{0}^{2}-1}(1+\right. & \log 2)) \\
& \geq \frac{1}{2^{4}} \frac{1}{\left(2^{\lambda(s)}-1\right)^{2}}
\end{aligned}
$$

Note that by $0 \leq \lambda(s) \leq 1$, we have $2^{\lambda(s)}-1 \leq \lambda(s)$. Thus, together with part 2 of Lemma B.1, the previous chain of inequalities implies

$$
\sum_{m=1}^{\left|J_{s_{0}}\right|-s_{0}^{2}} m \cdot 2^{-\lambda(s) m} \geq \frac{1}{2^{4}} \cdot\left|J_{s}\right|^{2} \geq \frac{1}{2^{6}} \cdot 2^{2 q(s+1)^{3}} .
$$

Together with (B.14), the inequality $\Xi \geq 2 \xi$, and our hypothesis $q \geq 100(\Xi+1)$, this implies

$$
\widehat{J}_{s_{0}}^{-}(\tau, \lambda(s)) \geq \frac{1}{2^{9}} 2^{2 q(s+1)^{3}-q \tau \cdot\left(s_{0}+1\right)^{3}-(\Xi+2 \xi) \tau \cdot\left(s_{0}+1\right)} .
$$

This proves part 1. 
2. Let $\varsigma$ be an integer in $\left[s_{0}-3, s_{0}\right]$ and note that by (B.1), (B.4), and the definition of $\widehat{J}_{\varsigma}^{-}$, we have

$$
\frac{J_{\varsigma}^{+}(\tau, \lambda(s))}{\widehat{J}_{\varsigma}^{-}(\tau, \lambda(s))}=2^{4 \tau \xi \cdot(\varsigma+1)+\lambda(s) \varsigma^{2}} \frac{\sum_{m=1}^{\left|J_{\varsigma}\right|} 2^{-\lambda(s) m}}{\sum_{m=1}^{\left|J_{\varsigma}\right|-\varsigma^{2}} m \cdot 2^{-\lambda(s) m}} .
$$

Suppose $\varsigma$ is in $\left[s_{0}-3, s_{0}-1\right]$. Then $\lambda(s)\left|J_{\varsigma}\right| \leq 1$, so

$$
\begin{aligned}
\frac{J_{\varsigma}^{+}(\tau, \lambda(s))}{\widehat{J}_{\varsigma}^{-}(\tau, \lambda(s))} & \leq 2 \cdot 2^{4 \tau \xi \cdot(\varsigma+1)+\lambda(s) \varsigma^{2}} \frac{\sum_{m=1}^{\left|J_{\varsigma}\right|} 2^{-\lambda(s) m}}{\sum_{m=1}^{\left|J_{\varsigma}\right|-\varsigma^{2}} m} \\
& \leq 2^{2} \cdot 2^{4 \tau \xi \cdot(\varsigma+1)+\lambda(s) \varsigma^{2}} \frac{\left|J_{\varsigma}\right|}{\left(\left|J_{\varsigma}\right|-\varsigma^{2}\right)^{2}} .
\end{aligned}
$$

Noting that by part 2 of Lemma B.1 and our hypotheses $s \geq 10$ and $q \geq 100(\Xi+1)$ we have

$$
\lambda(s) \varsigma^{2} \leq \varsigma^{2} /\left|J_{\varsigma}\right| \leq 1 \quad \text { and } \quad\left|J_{\varsigma}\right| \leq 2\left(\left|J_{\varsigma}\right|-\varsigma^{2}\right)
$$

By parts 2 and 3 of Lemma B.1 by the inequality $\Xi \geq 2 \xi$, by our hypotheses $q \geq$ $100(\Xi+1), \tau \in[1 / 2,1]$ and $s \geq 10$, we have

$$
\begin{aligned}
\left(b_{\varsigma}+\varsigma^{2}\right) \frac{J_{\varsigma}^{+}(\tau, \lambda(s))}{\widehat{J}_{\varsigma}^{-}(\tau, \lambda(s))} & \leq 2^{6} a_{\varsigma} 2^{4 \tau \xi \cdot(\varsigma+1)}\left|J_{\varsigma}\right|^{-1} \\
& \leq 2^{q \varsigma^{3}+q(\varsigma+1)-q(\varsigma+1)^{3}} \\
& \leq 2^{-q\left(3 \varsigma^{2}+2 \varsigma\right)} \\
& \leq 2^{-3 q \varsigma^{2}} \\
& \leq \frac{1}{20} 2^{-q s_{0}^{2}}
\end{aligned}
$$

This proves part 2 .

3. By part 3 of Lemma B.1 and the hypothesis $s \geq 10$ we have

$$
b_{s_{0}}-\left|J_{s_{0}-1}\right|+2 s_{0}^{2}=b_{s_{0}}-\left(a_{s_{0}}-b_{s_{0}-1}\right)+2 s_{0}^{2} \leq b_{s_{0}-1}+2 s_{0}^{2} \leq 2 \cdot 2^{q\left(s_{0}-1\right)^{3}} .
$$

Thus, by part 2 of Lemma B.1, (B.15), (B.16), and the inequality $\lambda(s) \leq 1$, we have

$$
\begin{aligned}
\left(b_{s_{0}}-\left|J_{s_{0}-1}\right|+2 s_{0}^{2}\right) \frac{J_{s_{0}}^{+}(\tau, \lambda(s))}{\widehat{J}_{s_{0}}^{-}(\tau, \lambda(s))} & \leq 2^{6} \cdot 2^{q\left(s_{0}-1\right)^{3}+4 \tau \xi \cdot\left(s_{0}+1\right)+\lambda(s) s_{0}^{2}}\left(2^{\lambda(s)}-1\right) \\
& \leq 2^{6} \lambda(s) \cdot 2^{q\left(s_{0}-1\right)^{3}+4 \tau \xi \cdot\left(s_{0}+1\right)+\lambda(s) s_{0}^{2}} \\
& \leq 2^{7} \cdot 2^{-q(s+1)^{3}+q\left(s_{0}-1\right)^{3}+4 \tau \xi \cdot\left(s_{0}+1\right)+\lambda(s) s_{0}^{2}} .
\end{aligned}
$$

Using $\lambda(s) s_{0}^{2}=s_{0}^{2}\left|J_{s}\right|^{-1} \leq 1$, the inequality $\Xi \geq 2 \xi$, and our hypotheses $q \geq$ $100(\Xi+1), \tau \in[1 / 2,1]$ and $s \geq 10$, we have

$$
\left(b_{s_{0}}-\left|J_{s_{0}-1}\right|+2 s_{0}^{2}\right) \frac{J_{s_{0}}^{+}(\tau, \lambda(s))}{\widehat{J}_{s_{0}}^{-}(\tau, \lambda(s))} \leq 2^{-q s_{0}^{3}+q\left(s_{0}-1\right)^{3}+q\left(s_{0}+1\right)} \leq \frac{1}{4} 2^{-q s_{0}^{2}} .
$$

This completes the proof of part 3 and of the lemma.

Proof of Lemma B.4 1. Note that for every $s \geq 0$, we have $\lambda(s) \leq 1$ and

$$
\sum_{m=1}^{+\infty} m \cdot 2^{-\lambda(s) m}=\frac{2^{\lambda(s)}}{\left(2^{\lambda(s)}-1\right)^{2}} \leq \frac{2^{\lambda(s)}}{(\lambda(s) \log 2)^{2}} \leq 2^{3}\left|J_{s}\right|^{2} \leq\left(2^{3}\right) 2^{2 q(s+1)^{3}} .
$$


Together with (B.1), (B.2), (B.3), for every $j$ in $\mathbb{N}_{0}$ this implies

$$
\begin{aligned}
\widetilde{J}_{j}^{+}(\tau, \lambda(s))+\widetilde{I}_{j+1}^{+}(\tau & , \lambda(s)) \\
& \leq 2^{-\tau\left(q(j+1)^{3}+\Xi \cdot(j+1)\right)+\tau \xi \cdot(2 j+3)} \sum_{k \in J_{j} \cup I_{j+1}} k \cdot 2^{-\lambda(s) k} \\
& \leq\left(2^{\tau \cdot \xi+3}\right) 2^{2 q(s+1)^{3}-q \tau \cdot(j+1)^{3}-\tau(\Xi-2 \xi) \cdot(j+1)} .
\end{aligned}
$$

By the inequality $\Xi-2 \xi \geq 1$ and our hypothesis that $\tau$ is in $[1 / 2,1]$, for every $j \geq$ $2 s+1$ we have

$$
\widetilde{J}_{j}^{+}(\tau, \lambda(s))+\widetilde{I}_{j+1}^{+}(\tau, \lambda(s)) \leq\left(2^{\xi+3}\right) 2^{-\tau \cdot(j+1)} .
$$

This implies that $\widetilde{\Pi}^{+}(\tau, \lambda(s))$ is finite, as wanted.

2. The first inequality follows directly from the definitions. Note that by (B.1) and (B.3), and our hypothesis that $\tau$ is in $[1 / 2,1]$, we have

$$
1+\widetilde{I}_{0}^{+}(\tau, \lambda(s)) \leq 1+\sum_{k=1}^{+\infty} k \cdot 2^{-\tau k+\xi}=1+2^{\xi} \frac{2^{-\tau}}{\left(1-2^{-\tau}\right)^{2}} \leq 10 \cdot 2^{\xi} .
$$

By part 3 of Lemma B.1 (B.1), (B.3), the inequality $\Xi-2 \xi \geq 1$ and our hypothesis that $\tau$ is in $[1 / 2,1]$, for every integer $j \geq 1$ we have

$$
\begin{aligned}
\widetilde{I}_{j}(\tau, \lambda(s)) & \leq\left(2^{\xi}\right) 2^{-\tau\left(q j^{3}+(\Xi-2 \xi) j\right)} \sum_{m=1}^{\left|I_{j}\right|}\left(a_{j}+m\right) 2^{-\tau m} \\
& \leq\left(2^{\xi} \cdot \frac{5}{4}\right) 2^{-\tau\left(q j^{3}+(\Xi-2 \xi) j\right)} a_{j} \sum_{m=1}^{\left|I_{j}\right|} 2^{-\tau m} \\
& \leq\left(2^{\xi+2}\right) 2^{q j^{3}-q \tau j^{3}-(\Xi-2 \xi) \tau j} \\
& \leq\left(2^{\xi+2}\right) 2^{q(1-\tau) j^{3}-\tau j} .
\end{aligned}
$$

Combined with (B.18) and our hypothesis that $\tau$ is in $[1 / 2,1]$, this implies

$$
1+\sum_{j=0}^{s_{0}+1} \widetilde{I}_{j}^{+}(\tau, \lambda(s)) \leq\left(2^{\xi+5}\right) 2^{(1-\tau) q\left(s_{0}+1\right)^{3}} .
$$

Together with part 1 of Lemma B.5, the inequality $\Xi \geq 2 \xi$ and our hypotheses $s \geq$ 10 and $q \geq 100(\Xi+1)$, this inequality implies

$$
1+\sum_{j=0}^{s_{0}+1} \widetilde{I}_{j}^{+}(\tau, \lambda(s)) \leq \frac{1}{2^{3}} 2^{-q s_{0}^{2}} \widehat{J}_{s_{0}}^{-}(\tau, \lambda(s)) .
$$

On the other hand, by (B.1) and (B.2) for every $j$ in $\left\{0, \ldots, s_{0}-4\right\}$ we have

$$
\begin{aligned}
\widetilde{J}_{j}^{+}(\tau, \lambda(s)) & =2^{-\tau\left(q(j+1)^{3}+\Xi \cdot(j+1)\right)+2 \tau \xi \cdot(j+1)} \sum_{k \in J_{j}} k \cdot 2^{-\lambda(s) k} \\
& \leq\left|J_{j}\right| 2^{q(j+1)^{3}-q \tau \cdot(j+1)^{3}-(\Xi-2 \xi) \tau \cdot(j+1)} \\
& \leq 2^{2 q(j+1)^{3}-q \tau \cdot(j+1)^{3}-(\Xi-2 \xi) \tau \cdot(j+1)} \\
& \leq 2^{2 q\left(s_{0}-3\right)^{3}-q \tau \cdot\left(s_{0}-3\right)^{3}-(\Xi-2 \xi) \tau \cdot\left(s_{0}-3\right)} .
\end{aligned}
$$


Together with part 1 of Lemma B.5, the inequality $\Xi \geq 2 \xi$ and with our hypotheses $q \geq 100(\Xi+1)$ and $s \geq 10$, this implies

$$
\frac{\sum_{j=0}^{s_{0}-4} \widetilde{J}_{j}^{+}(\tau, \lambda(s))}{\widehat{J}_{s_{0}}^{-}(\tau, \lambda(s))} \leq s_{0} 2^{-2 q s_{0}^{2}} \leq \frac{1}{2^{2}} 2^{-q s_{0}^{2}} .
$$

On the other hand, by (B.17), part 1 of Lemma B.5 and our hypotheses $q \geq$ $100(\Xi+1)$ and $\tau \in[1 / 2,1]$, for every integer $j \geq s_{0}+1$ we have

$$
\begin{aligned}
& \frac{\widetilde{J}_{j}^{+}(\tau, \lambda(s))+\widetilde{I}_{j+1}^{+}(\tau, \lambda(s))}{\widehat{J}_{s_{0}}^{-}(\tau, \lambda(s))} \\
& \quad \leq\left(2^{\xi+12}\right) 2^{-q \tau \cdot\left((j+1)^{3}-\left(s_{0}+1\right)^{3}\right)-(\Xi-2 \xi) \tau \cdot(j+1)+(\Xi+2 \xi) \tau \cdot\left(s_{0}+1\right)} \\
& \quad \leq\left(2^{\xi+12}\right) 2^{-q \tau \cdot\left(\left(j-s_{0}\right)^{3}+3\left(j-s_{0}\right)^{2}\left(s_{0}+1\right)+3\left(j-s_{0}\right)\left(s_{0}+1\right)^{2}\right)+4 \xi \tau\left(s_{0}+1\right)} \\
& \quad \leq \frac{1}{2^{4}} 2^{-q s_{0}^{2}\left(j-s_{0}\right)} .
\end{aligned}
$$

Summing over $j \geq s_{0}+1$ and using our hypotheses $q \geq 100(\Xi+1)$ and $s \geq 10$, we obtain

$$
\frac{\sum_{j=s_{0}+1}^{+\infty}\left(\widetilde{J}_{j}^{+}(\tau, \lambda(s))+\widetilde{I}_{j+1}^{+}(\tau, \lambda(s))\right)}{\widehat{J}_{s_{0}}^{-}(\tau, \lambda(s))} \leq \frac{1}{2^{4}} \frac{2^{-q s_{0}^{2}}}{1-2^{-q s_{0}^{2}}} \leq \frac{1}{2^{3}} 2^{-q s_{0}^{2}} .
$$

Combined with (B.19) and (B.20), this implies

$$
\widetilde{\Pi}^{+}(\tau, \lambda(s))-\sum_{\varsigma=s_{0}-3}^{s_{0}} \widetilde{J}_{\varsigma}^{+}(\tau, \lambda(s)) \leq \frac{1}{2} 2^{-q s_{0}^{2}} \widehat{J}_{s_{0}}^{-}(\tau, \lambda(s)) .
$$

Notice that for each positive integer $\varsigma$ we have

$$
\begin{aligned}
& \widetilde{J}_{\varsigma}^{+}(\tau, \lambda(s))-\widehat{J}_{\varsigma}^{+}(\tau, \lambda(s)) \\
& \leq \sum_{k=b_{\varsigma}}^{b_{\varsigma}+\varsigma^{2}-1} k \cdot 2^{-\lambda k-\tau N(k)+\tau \xi B(k)}+\sum_{k=b_{\varsigma}+\varsigma^{2}}^{a_{\varsigma}+1-1}\left(b_{\varsigma}+\varsigma^{2}\right) \cdot 2^{-\lambda k-\tau N(k)+\tau \xi B(k)} \\
& \leq\left(b_{\varsigma}+\varsigma^{2}\right) J_{\varsigma}^{+}(\tau, \lambda(s)) .
\end{aligned}
$$

Using this and part 2 of Lemma B.5 for each integer $\varsigma$ in $\left[s_{0}-3, s_{0}-1\right]$, we get

$$
\widetilde{J}_{\varsigma}^{+}(\tau, \lambda(s))-\widehat{J}_{\varsigma}^{+}(\tau, \lambda(s)) \leq \frac{1}{20} 2^{-q s_{0}^{2}} \widehat{J}_{\varsigma}^{-}(\tau, \lambda(s)) .
$$

Now by (B.22) with $s=s_{0}$ and part 3 of Lemma B.5 we have

$$
\begin{aligned}
\widetilde{J}_{s_{0}}^{+}(\tau, \lambda(s))-\widehat{J}_{s_{0}}^{+}(\tau, \lambda(s))-\left(\left|J_{s_{0}-1}\right|-s_{0}^{2}\right) J_{s_{0}}^{+}(\tau, \lambda(s)) & \leq \\
& \frac{1}{4} 2^{-q s_{0}^{2}} \widehat{J}_{s_{0}}^{-}(\tau, \lambda(s)) .
\end{aligned}
$$

Finally, putting together (B.21), (B.23) and (B.24) we obtain the desired inequality and complete the proof of the lemma. 


\section{REFERENCES}

[ADCS15] Michael Aizenman, Hugo Duminil-Copin, and Vladas Sidoravicius. Random currents and continuity of Ising model's spontaneous magnetization. Comm. Math. Phys., 334(2):719-742, 2015.

[BBS15] Viviane Baladi, Michael Benedicks, and Daniel Schnellmann. Whitney-Hölder continuity of the SRB measure for transversal families of smooth unimodal maps. Invent. Math., 201(3):773-844, 2015.

[BCFT17] K. Burns, V. Climenhaga, T. Fisher, and D. J. Thompson. Unique equilibrium states for geodesic flows in nonpositive curvature. ArXiv e-prints, March 2017.

[BGT18] Rodrigo Bissacot, Eduardo Garibaldi, and Philippe Thieullen. Zero-temperature phase diagram for double-well type potentials in the summable variation class. Ergodic Theory Dynam. Systems, 38(3):863-885, 2018.

[BL13] Henk Bruin and Renaud Leplaideur. Renormalization, thermodynamic formalism and quasi-crystals in subshifts. Comm. Math. Phys., 321(1):209-247, 2013.

[BL15] Henk Bruin and Renaud Leplaideur. Renormalization, freezing phase transitions and Fibonacci quasicrystals. Ann. Sci. Éc. Norm. Supér. (4), 48(3):739-763, 2015.

[BMS03] I. Binder, N. Makarov, and S. Smirnov. Harmonic measure and polynomial Julia sets. Duke Math. J., 117(2):343-365, 2003.

[Bow75] Rufus Bowen. Equilibrium states and the ergodic theory of Anosov diffeomorphisms. Lecture Notes in Mathematics, Vol. 470. Springer-Verlag, Berlin, 1975.

[BTT17] H. Bruin, D. Terhesiu, and M. Todd. The pressure function for infinite equilibrium measures. ArXiv e-prints, November 2017.

[CG93] Lennart Carleson and Theodore W. Gamelin. Complex dynamics. Universitext: Tracts in Mathematics. Springer-Verlag, New York, 1993.

[CH10] Jean-René Chazottes and Michael Hochman. On the zero-temperature limit of Gibbs states. Comm. Math. Phys., 297(1):265-281, 2010.

[Cli18] Vaughn Climenhaga. Specification and towers in shift spaces. Comm. Math. Phys., 364(2):441-504, 2018.

[CR17] D. Coronel and J. Rivera-Letelier. Sensitive dependence of geometric Gibbs states. ArXiv e-prints, August 2017.

[CRL13] Daniel Coronel and Juan Rivera-Letelier. Low-temperature phase transitions in the quadratic family. Adv. Math., 248:453-494, 2013.

[CRL15a] Daniel Coronel and Juan Rivera-Letelier. High-order phase transitions in the quadratic family. J. Eur. Math. Soc. (JEMS), 17(11):2725-2761, 2015.

[CRL15b] Daniel Coronel and Juan Rivera-Letelier. Sensitive dependence of Gibbs measures at low temperatures. J. Stat. Phys., 160(6):1658-1683, 2015.

[CRT16] Y. Moo Chung, J. Rivera-Letelier, and H. Takahasi. Large deviation principle in onedimensional dynamics. ArXiv e-prints, October 2016.

[DGR14] L. J. Díaz, K. Gelfert, and M. Rams. Abundant rich phase transitions in step-skew products. Nonlinearity, 27(9):2255-2280, 2014.

[DH84] A. Douady and J. H. Hubbard. Étude dynamique des polynômes complexes. Partie I, volume 84 of Publications Mathématiques d'Orsay [Mathematical Publications of Orsay]. Université de Paris-Sud, Département de Mathématiques, Orsay, 1984.

[DH85] Adrien Douady and John Hamal Hubbard. On the dynamics of polynomial-like mappings. Ann. Sci. École Norm. Sup. (4), 18(2):287-343, 1985.

[dMvS93] Welington de Melo and Sebastian van Strien. One-dimensional dynamics, volume 25 of Ergebnisse der Mathematik und ihrer Grenzgebiete (3) [Results in Mathematics and Related Areas (3)]. Springer-Verlag, Berlin, 1993.

[Dob12] Neil Dobbs. Measures with positive Lyapunov exponent and conformal measures in rational dynamics. Trans. Amer. Math. Soc., 364(6):2803-2824, 2012.

[Dob15] Neil Dobbs. Pesin theory and equilibrium measures on the interval. Fund. Math., 231(1):1-17, 2015.

[GPR10] Katrin Gelfert, Feliks Przytycki, and Michał Rams. On the Lyapunov spectrum for rational maps. Math. Ann., 348(4):965-1004, 2010.

[GPR16] Katrin Gelfert, Feliks Przytycki, and Michał Rams. Lyapunov spectrum for multimodal maps. Ergodic Theory Dynam. Systems, 36(5):1441-1493, 2016. 
[Her07] Igor Herbut. A modern approach to critical phenomena. Cambridge University Press, Cambridge, 2007.

[Klo17] B. Kloeckner. An optimal transportation approach to the decay of correlations for non-uniformly expanding maps. ArXiv e-prints, November 2017.

[KN92] Gerhard Keller and Tomasz Nowicki. Spectral theory, zeta functions and the distribution of periodic points for Collet-Eckmann maps. Comm. Math. Phys., 149(1):31-69, 1992.

[LRL14a] Huaibin Li and Juan Rivera-Letelier. Equilibrium states of interval maps for hyperbolic potentials. Nonlinearity, 27(8):1779-1804, 2014.

[LRL14b] Huaibin Li and Juan Rivera-Letelier. Equilibrium states of weakly hyperbolic onedimensional maps for Hölder potentials. Comm. Math. Phys., 328(1):397-419, 2014.

[Mil00] John Milnor. Periodic orbits, externals rays and the Mandelbrot set: an expository account. Astérisque, (261):xiii, 277-333, 2000. Géométrie complexe et systèmes dynamiques (Orsay, 1995).

[Mil06] John Milnor. Dynamics in one complex variable, volume 160 of Annals of Mathematics Studies. Princeton University Press, Princeton, NJ, third edition, 2006.

[NS98] Tomasz Nowicki and Duncan Sands. Non-uniform hyperbolicity and universal bounds for S-unimodal maps. Invent. Math., 132(3):633-680, 1998.

[OW17] H. Oh and D. Winter. Prime number theorems and holonomies for hyperbolic rational maps. Inventiones Mathematicae, 208:401-440, May 2017.

[PR14] F. Przytycki and J. Rivera-Letelier. Geometric pressure for multimodal maps of the interval. Mem. Amer. Math. Soc., to appear, ArXiv e-prints, May 2014.

[PRL11] Feliks Przytycki and Juan Rivera-Letelier. Nice inducing schemes and the thermodynamics of rational maps. Comm. Math. Phys., 301(3):661-707, 2011.

[PRLS03] Feliks Przytycki, Juan Rivera-Letelier, and Stanislav Smirnov. Equivalence and topological invariance of conditions for non-uniform hyperbolicity in the iteration of rational maps. Invent. Math., 151(1):29-63, 2003.

[Prz18] F. Przytycki. Thermodynamic formalism methods in one-dimensional real and complex dynamics. In Proc. Int. Cong. of Math., pages 2081-2106, 2018.

[Riv12] J. Rivera-Letelier. Asymptotic expansion of smooth interval maps. ArXiv e-prints, April 2012.

[Roe00] Pascale Roesch. Holomorphic motions and puzzles (following M. Shishikura). In The Mandelbrot set, theme and variations, volume 274 of London Math. Soc. Lecture Note Ser., pages 117-131. Cambridge Univ. Press, Cambridge, 2000.

[Rue76] David Ruelle. A measure associated with axiom-A attractors. Amer. J. Math., 98(3):619-654, 1976.

[Sin72] Ja. G. Sină. Gibbs measures in ergodic theory. Uspehi Mat. Nauk, 27(4(166)):21-64, 1972.

[Vel17] A. Velozo. Phase transitions for geodesic flows and the geometric potential. ArXiv e-prints, April 2017.

[vER07] A. C. D. van Enter and W. M. Ruszel. Chaotic temperature dependence at zero temperature. J. Stat. Phys., 127(3):567-573, 2007.

[VV10] Paulo Varandas and Marcelo Viana. Existence, uniqueness and stability of equilibrium states for non-uniformly expanding maps. Ann. Inst. H. Poincaré Anal. Non Linéaire, 27(2):555-593, 2010.

Daniel Coronel, Departamento de Matemáticas, Universidad Andrés Bello, Avenida República 220, Santiago, Chile

E-mail address: alvaro.coronel@unab.cl

Department of Mathematics, University of Rochester. Hylan Building, Rochester, NY 14627, U.S.A.

E-mail address: riveraletelier@gmail.com

$U R L:$ http://rivera-letelier.org/ 